\title{
Optimal liquidation trajectories for the Almgren-Chriss model
}

\author{
Arne Løkka* $\quad$ Junwei $\mathrm{Xu}^{\dagger}$
}

October 12, 2020

\begin{abstract}
We consider an optimal liquidation problem with infinite horizon in the Almgren-Chriss framework, where the unaffected asset price follows a Lévy process. The temporary price impact is described by a general function that satisfies some reasonable conditions. We consider a market agent with constant absolute risk aversion, who wants to maximize the expected utility of the cash received from the sale of the agent's assets, and show that this problem can be reduced to a deterministic optimization problem that we are able to solve explicitly. In order to compare our results with exponential Lévy models, which provide a very good statistical fit with observed asset price data for short time horizons, we derive the (linear) Lévy process approximation of such models. In particular we derive expressions for the Lévy process approximation of the exponential variance-gamma Lévy process, and study properties of the corresponding optimal liquidation strategy. We then provide a comparison of the liquidation trajectories for reasonable parameters between the Lévy process model and the classical Almgren-Chriss model. In particular, we obtain an explicit expression for the connection between the temporary impact function for the Lévy model and the temporary impact function for the Brownian motion model (the classical Almgren-Chriss model), for which the optimal liquidation trajectories for the two models coincide.
\end{abstract}

Keywords: Lévy processes, Almgren-Chriss model, algorithmic trading, optimal liquidation, optimal execution, constant absolute risk aversion, market impact, optimal control, Hamilton-Jacobi-Bellman equation

\section{Introduction}

The introduction of electronic trading platforms was followed by an increased interest in how to split large orders into smaller orders in order to liquidate large asset positions. An important question for market agents is how to sell a large number of shares. Because of a

*Department of Mathematics Columbia House London School of Economics Houghton Street, London WC2A 2AE United Kingdom (a.lokka@lse.ac.uk)

${ }^{\dagger}$ Department of Mathematics Columbia House London School of Economics Houghton Street, London WC2A 2AE United Kingdom (j.xu19@lse.ac.uk) 
lack of liquidity in the market it is often not practical to sell all the shares immediately since this can result in too high an execution cost. By splitting a large block of orders into smaller ones, the agent can often reduce the cost substantially. The problem of finding the optimal way to do this has therefore been the subject of considerable interest.

When the agent determines the speed at which to sell the shares, the key components are execution cost and market risk. A slow execution speed will result in a low execution cost, but high market risk. On the other hand, a rapid execution speed will result in a low market risk, but high execution cost. In most models dealing with optimal execution, Brownian motion is driving the market risk. However, in reality observed stock price data demonstrate that Brownian motion is not a particularly good model for stock prices, especially for shorter time periods. For instance, sudden large price movements and the heavy-tailed distributions of logreturns can not be captured by Brownian motion. Also, observed logarithmic stock returns over short time-horizons are not normally distributed. On the other hand, there are many theoretical and empirical studies that show that Lévy processes provide a good fit to market data. For detailed discussions, we refer to Madan and Seneta (1990), Eberlein and Keller (1995) and Barndorff-Nielsen (1997). For the reasons explained above, that in practise the time it takes to liquidate is often very short and that Lévy processes provide good statistical models for stock prices over short periods, we shall consider models based on Lévy processes.

We consider a continuous-time optimal liquidation problem of a single stock in the AlmgrenChriss framework with infinite time horizon. The permanent impact function is supposed to be linear, and we describe the temporary market impact function in terms of general sufficient conditions ensuring that we are able to solve the problem explicitly. The unaffected share price is driven by a linear Lévy process. We assume that the market agent is not permitted to buy back shares during liquidation, but we can in any case show by a dynamic programming argument that any such strategy would be sub-optimal. The agent is supposed to have constant absolute risk aversion (CARA), and the aim is to maximize expected utility of the final cash position over a set of admissible liquidation strategies. Following an idea introduced in Schied et al. (2010), the optimization problem is reduced to an optimization problem over a set of deterministic strategies. Moreover, we show that for a general Lévy process, there is no immediate relationship between the optimal strategy for the mean-variance criteria and the optimal strategy for the expected exponential utility, which holds for the Brownian motion case. We also show that when the Lévy process is a strict submartingale, our problem is illposed, and it is always optimal to hold on to the shares rather than sell. Then by solving the Hamilton-Jacobi-Bellman equation, the optimal liquidation strategy is derived in an explicit form. After that, we provide some conditions that determine whether the optimal strategy has a finite termination time.

The standard way to analyze stock price data is to find the statistics of the log-returns. This naturally leads to exponential Lévy models, and most distributions for the driving Lévy process in relation to stock price data are of the exponential model type. Given a specific exponential Lévy model, we therefore show how to linearize the model in order to get a model of the form relevant to our paper. We then provide some examples where we assume the 
log-returns of the share price satisfy the variance-gamma distribution and where they satisfy the normal distribution. In the variance-gamma case we find that the widely used power law market impact function can result in optimal strategies which liquidate faster than what seems practical. We point out that the cost from large trading speeds may be underestimated by power functions, and that a function with a bigger growth rate may better reflect the cost of execution.

For an introduction to high-frequency trading and optimal execution, we refer the reader to Lehalle and Laruelle (2013), Cartea et al. (2015) and Guéant (2016), but below we provide a brief review of the more relevant works in connection to this paper. Bertsimas and Lo (1998) introduced a discrete time stock price model with illiquidity effects and related problems. Then Almgren and Chriss $(1999,2000)$ classified the effects in terms of permanent and temporary impacts of trading. In this kind of market impact framework, various liquidation models were developed. Almgren and Chriss (2000) introduced a discrete-time model with linear permanent and temporary impact functions, a deterministic optimal trading strategy was derived by mean-variance optimization. Almgren (2003) generalized the model by considering non-linear impact functions. A single-asset continuous model with infinite time horizon was introduced in Schied and Schöneborn (2009), a multi-asset finite horizon model was considered by Schied et al. (2010) and Schöneborn (2016) provides a multi-asset infinite horizon model. In these papers strategies were derived by maximizing expected utilities instead of the meanvariance criteria. Schied et al. (2010) explained the relationship between the mean-variance criteria and the expected exponential utility criteria in the Almgren-Chriss framework. They also proved that in a finite time horizon, when the stock price is driven by a Lévy process and the agent has exponential utility, the optimal strategy is deterministic. Gatheral (2010) suggested that instead of dealing with permanent and temporary impacts, the market impact should decay over time. Moreover, Obizhaeva and Wang (2013) introduced a limit order book model and calculated the optimal execution strategy for such a model. Afterwards, several authors considered variations of this limit order book model, such as Alfonsi and Schied (2010), Alfonsi et al. (2010), Alfonsi et al. (2012) and Løkka (2014). In the literature of continuous models of optimal execution, price processes are often linear and impact additive. However, by considering a new optimization criterion, a model in the Almgren-Chriss framework based on geometric Brownian motion with linear market impact was given in Gatheral and Schied (2011). Then Schied (2013) extended this model to general square integrable semimartingales. Also, some multiplicative impact models are introduced in Forsyth et al. (2012) and Guo and Zervos (2015). In particular, Forsyth et al. (2012) demonstrated that the linear model gives an excellent approximation to models with prices modelled as a geometric Brownian motion and multiplicative impact in the Almgren-Chriss framework.

The structure of this paper is the following. In Section 2 we introduce the model and the optimal execution problem. We reduce the problem to a deterministic optimization problem in Section 3, and solve it in Section 4. In Section 5 we show how to linearize exponential Lévy models, and illustrate with examples in Section 6. We then summarize our results and briefly point out some directions for future research in section 7 . 


\section{Problem formulation}

Let $(\Omega, \mathcal{F}, \mathbb{P})$ be a complete probability space, equipped with a filtration $\mathbb{F}=\left(\mathcal{F}_{t}\right)_{t \geq 0}$ satisfying the usual conditions, which supports a one dimensional, non-trivial, $\mathbb{F}$-adapted Lévy process $L$. We assume that the Lévy process $L$ possesses the following properties.

Assumption 2.1. $L_{1}$ has finite second moment. Moreover, the set $\left\{\delta<0 \mid \mathbb{E}\left[\mathrm{e}^{\delta L_{1}}\right]<\infty\right\}$ is non-empty.

For future reference, we observe that this assumption ensures that $L_{t}$ has finite first and second moments, for all $t \geq 0$. Hence, $L$ admits the decomposition

$$
L_{t}=\mu t+\sigma W_{t}+\int_{\mathbb{R}} x(N(t, d x)-t \nu(d x)),
$$

where $\mu \in \mathbb{R}$ and $\sigma \geq 0$ are two constants, $W$ is a standard Brownian motion, $N$ is a Poisson random measure which is independent of $W$ with compensator $t \nu(d x)$, and $\nu$ is the Lévy measure associated with $L$ Kyprianou, 2006). Set

$$
\bar{\delta}=\inf \left\{\delta<0 \mid \mathbb{E}\left[\mathrm{e}^{\delta L_{1}}\right]<\infty\right\}<0 .
$$

Then Assumption 2.1 also ensures that the cumulant generating function of $L_{1}$ is finite on the interval $(\bar{\delta}, 0]$.

We consider a market agent (e.g. an institutional trader or corporation) intending to sell a large number of shares of a single stock. For $t \geq 0$, we denote by $Y_{t}$ the agent's position in the stock at time $t$, and let $y \geq 0$ denote the agent's initial stock position. We consider the following sets of admissible liquidation strategies.

Definition 2.2. Given an initial share position $y \geq 0$, the set of admissible strategies, denoted by $\mathcal{A}(y)$, consists of all $\mathbb{F}$-adapted, absolutely continuous, non-increasing processes $Y$ satisfying

$$
\int_{0}^{\infty}\left\|Y_{t}\right\|_{L^{\infty}(\mathbb{P})} d t<\infty \quad \text { if } \mu \neq 0
$$

and

$$
\int_{0}^{\infty}\left\|Y_{t}\right\|_{L^{\infty}(\mathbb{P})}^{2} d t<\infty \quad \text { if } \mu=0 .
$$

Let $\mathcal{A}_{D}(y)$ be the set of all deterministic strategies in $\mathcal{A}(y)$.

The reason for operating with different sets of admissibility depending on the drift parameter $\mu$ is related to the asymptotic properties of the cumulant generating function of $L_{1}$ around 0 . If $\mu$ is 0 then the cumulant generating function is of order two around zero, while it is of order one if $\mu$ is different from zero (the importance of the cumulant generating function of $L_{1}$ will be explained later). The integrability conditions in (2.3) and (2.4) guarantee that 
the agent's finial cash position is well-defined (see Proposition 2.6), and are also necessary in order for the optimization problem to be well defined (see Remark 2.7).

Let $Y \in \mathcal{A}(y)$. Then there exists an $\mathbb{F}$-adapted, positive-valued process $\xi$ such that $Y$ admits the representation

$$
Y_{t}=y-\int_{0}^{t} \xi_{s} d s
$$

i.e. $-\xi_{t}$ is the time derivative of $Y$ at time $t$. In the literature of optimal liquidation, the function $t \mapsto Y_{t}$ is referred to as the liquidation trajectory and the associated process $\xi$ as the liquidation speed (Almgren and Chriss, 2000, (Almgren, 2003).

It is common in the optimal liquidation literature to refer to the price process observed in the market if the agent does not trade as the unaffected stock price process. Throughout this chapter we assume that the unaffected stock price process is modelled by

$$
s+L_{t}, \quad t \geq 0
$$

where $s>0$ is some constant which denotes the initial stock price. In reality, liquidation is often completed in a very short time. It is well known that Lévy processes provide a good fit of the observed stock returns over short time horizons. Therefore the model should provide a good balance between the the cost of liquidating the position and the corresponding market risk. Following Almgren and Chriss (1999, 2000) and Almgren (2003), we split market impact into two components: a permanent impact and a temporary impact. We therefore assume that the stock price at time $t \geq 0$ is given by

$$
S_{t}=s+L_{t}+\alpha\left(Y_{t}-Y_{0}\right)-F\left(\xi_{t}\right)
$$

where $\alpha \geq 0$ is a constant describing the permanent impact and $F:[0, \infty) \rightarrow[0, \infty)$ is a function describing the temporary impact. We assume that $F$ satisfies the following assumptions.

Assumption 2.3. The temporary impact function $F:[0, \infty) \rightarrow[0, \infty)$ satisfies that

(i) $F \in C([0, \infty)) \cap C^{1}((0, \infty))$,

(ii) $F(0)=0$,

(iii) the function $x \mapsto x F(x)$ is strictly convex on $[0, \infty)$,

(iv) the function $x \mapsto x^{2} F^{\prime}(x)$ is strictly increasing, and it tends to infinity as $x \rightarrow \infty$.

In the above assumption, condition (iii) serves to ensure convexity of the objective function in the optimization problem we are going to solve (see (3.27)) and hence uniqueness of the solution (see Theorem 4.3), condition (iv) ensures that the value function in our optimization problem is solved in an explicit form (see Proposition 4.2) and that the optimal liquidation 
speed process can be expressed in a feedback form (see Theorem 4.3). Assumption 2.3 is satisfied by a large class of functions, for example $F(x)=\beta x^{\gamma}$ with $\beta, \gamma>0$ or

$$
F(x)= \begin{cases}\beta_{1} x^{0.6} & x \in[0, \bar{x}], \\ \beta_{2} e^{\gamma(x-\bar{x}+\hat{x})}-\beta_{2} e^{\gamma \hat{x}}+\beta_{1} \bar{x}^{0.6} & x \in(\bar{x}, \infty),\end{cases}
$$

where $\beta_{1}, \beta_{2}, \gamma$ and $\bar{x}$ are strictly positive constants and $\hat{x}$ is given by

$$
\hat{x}=\frac{\ln \left(\frac{3 \beta_{1}}{5 \beta_{2} \gamma}\right)-\frac{2}{5} \ln \bar{x}}{\gamma} .
$$

Under this assumption, we derive the following technical properties of $F$ for future reference.

Lemma 2.4. $F$ is strictly increasing and $\lim _{x \rightarrow 0} x F^{\prime}(x)=0$. Hence $\lim _{x \rightarrow 0} x^{2} F^{\prime}(x)=0$.

Proof. For $\lambda \in(0,1)$ and $x \in(0, \infty)$, Assumption 2.3 (ii) and (iii) imply that $F(\lambda x)<$ $\lambda F(x)<F(x)$, which shows that $F$ is strictly increasing.

The derivative of $x \mapsto x F(x)$, together with the convexity of this function, implies that $\lim _{x \rightarrow 0} x F^{\prime}(x)$ exists. As $F^{\prime}(x)>0$, for all $x>0$, it follows that $\lim _{x \rightarrow 0} x F^{\prime}(x) \geq 0$. Suppose $\lim _{x \rightarrow 0} x F^{\prime}(x)>0$. Then there exist constants $\bar{x}>0$ and $c>0$, such that for all $x \in(0, \bar{x})$,

$$
F^{\prime}(x)>\frac{c}{x} \text {. }
$$

But then,

$$
F(\bar{x})=\lim _{x \rightarrow 0} \int_{x}^{\bar{x}} F^{\prime}(u) d u \geq \lim _{x \rightarrow 0} \int_{x}^{\bar{x}} \frac{c}{u} d u=\infty,
$$

which contradicts the continuity of $F$. Hence, $\lim _{x \rightarrow 0} x F^{\prime}(x)=0$, and it therefore follows that $\lim _{x \rightarrow 0} x^{2} F^{\prime}(x)=0$.

For $t \geq 0$, let $C_{t}^{Y}$ denote the cash position of the agent at time $t$ associated with an admissible strategy $Y$. Denote by $c \in \mathbb{R}$ the agent's initial cash position. Then a direct calculation verifies that the cash position at some finite time $T$ is given by

$$
\begin{aligned}
C_{T}^{Y} & =c-\int_{0}^{T} S_{t} d Y_{t} \\
& =c-(s-\alpha y)\left(Y_{T}-y\right)+\frac{\alpha}{2}\left(y^{2}-Y_{T}^{2}\right)-L_{T} Y_{T}+\int_{0}^{T} Y_{t-} d L_{t}-\int_{0}^{T} \xi_{t} F\left(\xi_{t}\right) d t .
\end{aligned}
$$

The next lemma is used in the proof of Proposition 2.6, which states that the agent's cash position at the end of time is well-defined.

Lemma 2.5. Let $Z$ be a positive-valued, decreasing process satisfying $\int_{0}^{\infty} Z_{t} d t<\infty$. Then $t Z_{t} \rightarrow 0$, as $t \rightarrow \infty$. 
Proof. Suppose $\liminf _{t \rightarrow \infty} t Z_{t}>0$, then there exists some constant $c$ such that

$$
\liminf _{t \rightarrow \infty} t Z_{t}>c>0
$$

This implies that we can find some $s \geq 0$ such that for all $t \geq s$,

$$
Z_{t}>\frac{c}{t}
$$

Hence

$$
\int_{s}^{\infty} Z_{t} d t \geq \int_{s}^{\infty} \frac{c}{t} d t=\infty
$$

which contradicts $\int_{0}^{\infty} Z_{t} d t<\infty$. Thus, we have shown that

$$
\liminf _{t \rightarrow \infty} t Z_{t}=0
$$

We know that $Z$ is a decreasing process, which is of finite variation. By Itô's formula we calculate that

$$
t Z_{t}=\int_{0}^{t} u d Z_{u}+\int_{0}^{t} Z_{u} d u
$$

It can be observed that $t \mapsto \int_{0}^{t} u d Z_{u}$ is negative and decreasing while $t \mapsto \int_{0}^{t} Z_{u} d u$ is positive and increasing. Then,

$$
0 \leq \sup _{t \geq r} t Z_{t} \leq \sup _{t \geq r} \int_{0}^{t} u d Z_{u}+\sup _{t \geq r} \int_{0}^{t} Z_{u} d u=\int_{0}^{r} u d Z_{u}+\int_{0}^{\infty} Z_{u} d u .
$$

Also,

$$
\inf _{t \geq r} t Z_{t} \geq \inf _{t \geq r} \int_{0}^{t} u d Z_{u}+\inf _{t \geq r} \int_{0}^{t} Z_{u} d u=\int_{0}^{\infty} u d Z_{u}+\int_{0}^{r} Z_{u} d u
$$

Taking $r$ to infinity in $(2.19)$ and $(2.18)$, and by (2.16) we have

$$
\begin{aligned}
& 0 \leq \limsup _{t \rightarrow \infty} t Z_{t}=\lim _{r \rightarrow \infty} \sup _{t \geq r} t Z_{t} \leq \int_{0}^{\infty} u d Z_{u}+\int_{0}^{\infty} Z_{u} d u, \\
& 0=\liminf _{t \rightarrow \infty} t Z_{t}=\liminf _{r \rightarrow \infty} \operatorname{in}_{t \geq r} t Z_{t} \geq \int_{0}^{\infty} u d Z_{u}+\int_{0}^{\infty} Z_{u} d u .
\end{aligned}
$$

Therefore, we conclude that $\lim _{t \rightarrow \infty} t Z_{t}=0$.

Proposition 2.6. For any $Y \in \mathcal{A}(y)$, we have

(i) $L_{T} Y_{T} \rightarrow 0$ in $L^{2}(\mathbb{P})$, as $T \rightarrow \infty$, 
(ii) $\int_{0}^{\infty} Y_{t-} d L_{t}$ is well-defined in $L^{1}(\mathbb{P})$.

Therefore,

$$
C_{\infty}^{Y}=c+s y-\frac{1}{2} \alpha y^{2}+\int_{0}^{\infty} Y_{t-} d L_{t}-\int_{0}^{\infty} \xi_{t} F\left(\xi_{t}\right) d t, \quad \text { a.s. }
$$

for any $Y \in \mathcal{A}(y)$.

Proof. Let $f$ be the characteristic function of $L_{t}$, so

$$
f(u)=\mathbb{E}\left[\mathrm{e}^{\mathrm{i} u L_{t}}\right]=\mathrm{e}^{t \psi(u)},
$$

where $\psi(u)$ is given by the Lévy-Khintchine representation of $L$. By Assumption 2.1 we know that $f$, hence $\psi$, are twice differentiable at 0 . Hence, we calculate that $f^{\prime}(0)=\mathrm{i} \mathbb{E}\left[L_{t}\right]=t \psi^{\prime}(0)$ and $f^{\prime \prime}(0)=-\mathbb{E}\left[L_{t}^{2}\right]$, and therefore,

$$
\mathbb{E}\left[L_{t}^{2}\right]=(\mu t)^{2}-\psi^{\prime \prime}(0) t
$$

Then,

$$
\mathbb{E}\left[\left(L_{t} Y_{t}\right)^{2}\right] \leq \mathbb{E}\left[L_{t}^{2}\right]\left\|Y_{t}\right\|_{L^{\infty}(\mathbb{P})}^{2}=\mu^{2}\left(t\left\|Y_{t}\right\|_{L^{\infty}(\mathbb{P})}\right)^{2}-\psi^{\prime \prime}(0) t\left\|Y_{t}\right\|_{L^{\infty}(\mathbb{P})}^{2} .
$$

If $\mu \neq 0$, then for any $Y \in \mathcal{A}(y),\left(\left\|Y_{t}\right\|_{L^{\infty}(\mathbb{P})}\right)_{t \geq 0}$ and $\left(\left\|Y_{t}\right\|_{L^{\infty}(\mathbb{P})}^{2}\right)_{t \geq 0}$ are continuous, positive and decreasing. The integrability condition in $(2.3)$ implies that $\int_{0}^{\infty}\left\|Y_{t}\right\|_{L^{\infty}(\mathbb{P})}^{2} d t<\infty$. Therefore, according to Lemma 2.5 we have

$$
\lim _{t \rightarrow \infty} t\left\|Y_{t}\right\|_{L^{\infty}(\mathbb{P})}=0 \quad \text { and } \quad \lim _{t \rightarrow \infty} t\left\|Y_{t}\right\|_{L^{\infty}(\mathbb{P})}^{2}=0 .
$$

Hence, by (2.25) and the finiteness of $\mu$ and $\psi^{\prime \prime}(0)$ we conclude that

$$
\lim _{T \rightarrow \infty} \mathbb{E}\left[\left(L_{t} Y_{t}\right)^{2}\right]=0
$$

When $\mu=0$, we get $\int_{0}^{\infty}\left\|Y_{t}\right\|_{L^{\infty}(\mathbb{P})}^{2} d t<\infty$ directly as a condition of admissible strategies. Therefore, the same result follows. 
It remains to prove part (ii). Using Cauchy-Schwarz inequality and Itô isometry we obtain

$$
\begin{aligned}
& \mathbb{E}\left[\left|\int_{0}^{T} Y_{t-} d L_{t}\right|\right] \\
\leq & |\mu| \mathbb{E}\left[\left|\int_{0}^{T} Y_{t-} d t\right|\right]+\mathbb{E}\left[\left|\int_{0}^{T} Y_{t-} d\left(\sigma W_{t}+\int_{\mathbb{R}} x(N(t, d x)-t \nu(d x))\right)\right|\right] \\
\leq & |\mu| \int_{0}^{T}\left\|Y_{t}\right\|_{L^{\infty}(\mathbb{P})} d t+\mathbb{E}\left[\left|\int_{0}^{T} Y_{t-} d\left(\sigma W_{t}+\int_{\mathbb{R}} x(N(t, d x)-t \nu(d x))\right)\right|^{2}\right]^{\frac{1}{2}} \\
= & |\mu| \int_{0}^{T}\left\|Y_{t}\right\|_{L^{\infty}(\mathbb{P})} d t+\left(\sigma^{2}+\int_{\mathbb{R} \backslash\{0\}} x^{2} \nu(d x)\right)^{\frac{1}{2}} \mathbb{E}\left[\int_{0}^{T} Y_{t}^{2} d t\right]^{\frac{1}{2}} \\
\leq & |\mu| \int_{0}^{T}\left\|Y_{t}\right\|_{L^{\infty}(\mathbb{P})} d t+\left(\sigma^{2}+\int_{\mathbb{R} \backslash\{0\}} x^{2} \nu(d x)\right)^{\frac{1}{2}} \mathbb{E}\left[\int_{0}^{T}\left\|Y_{t}\right\|_{L^{\infty}(\mathbb{P})}^{2} d t\right]^{\frac{1}{2}}
\end{aligned}
$$

From the existence of the first and second moments of $L_{1}$, we know that $\mu, \sigma$ and $\int_{\mathbb{R} \backslash\{0\}} x^{2} \nu(d x)$ are all finite. The result then follows from the integrability conditions in (2.3) and (2.4) of an admissible strategy.

From the expression of $C_{\infty}^{Y}$, we can make a few observations. The term $c+s y$ can be viewed as the initial mark-to-market wealth of the agent. The agent's total loss due to the permanent impact of trading is given by $\frac{1}{2} \alpha y^{2}$, which is deterministic and only depends on the initial liquidation size. In particular, it does not depend on the choice of liquidation strategy. The term $\int_{0}^{\infty} \xi_{t} F\left(\xi_{t}\right) d t$ represents the total cost due to the temporary impact, and it does depend on the liquidation strategy. The term $\int_{0}^{\infty} Y_{t-} d L_{t}$ represents the gain or loss due to market volatility. A relatively slow liquidation speed reduces the temporary impact, but provides a substantial market volatility risk. The optimal liquidation strategy is therefore a compromise between the loss due to the temporary impact and the market volatility risk. We assume that the agent has a constant absolutely risk aversion (CARA), thus the agent's utility function $U$ satisfies $U(x)=-\exp (-A x)$, for some constant $A>0$. The agent aims to maximize the expected utility of the cash position at the end of time, i.e. the agent wants to solve

$$
\sup _{Y \in \mathcal{A}(y)} \mathbb{E}\left[U\left(C_{\infty}^{Y}\right)\right]
$$

In view of $(2.22)$, this problem takes the form of

$$
\inf _{Y \in \mathcal{A}(y)} \mathrm{e}^{-A \widetilde{C}} \mathbb{E}\left[\exp \left(-\int_{0}^{\infty} A Y_{t-} d L_{t}+A \int_{0}^{\infty} \xi_{t} F\left(\xi_{t}\right) d t\right)\right],
$$


where

$$
\widetilde{C}=c+s y-\frac{1}{2} \alpha y^{2} .
$$

To solve the above problem, it is sufficient to look at

$$
\inf _{Y \in \mathcal{A}(y)} \mathbb{E}\left[\exp \left(-\int_{0}^{\infty} A Y_{t-} d L_{t}+A \int_{0}^{\infty} \xi_{t} F\left(\xi_{t}\right) d t\right)\right] .
$$

Remark 2.7. Suppose that we do not impose integrability conditions (2.3) and (2.4) on an admissible strategy. The cash position at time infinity may then not be well-defined, but one may consider to solve the problem

$$
\sup _{Y \in \mathcal{A}(y)} \mathbb{E}\left[-\exp \left(-A \limsup _{T \rightarrow \infty} C_{T}^{Y}\right)\right] .
$$

However, without (2.3) and (2.4), our model admits an arbitrage in some weak sense. To see this, take for instance the Lévy process $L$ to be a standard Brownian motion and consider some stock price $p>s$. Write $\tau_{p}=\inf \left\{t \geq 0 \mid L_{t} \geq p\right\}$ which is finite a.s. (by Lemma 3.6 in Rogers and Williams (2000)). Suppose $Y^{p}$ is an absolutely continuous, non-increasing strategy which consists of waiting until time $\tau_{p}$ and then decreases to 0 in a deterministic way during a finite time $T_{p}$, i.e. $\left(Y_{\tau_{p}+t}^{p}\right)_{0 \leq t \leq T_{p}}$ is a deterministic process starting from $p$ with $Y_{\tau_{p}+T_{p}}^{p}=0$. Let $\xi^{p}$ be the associated speed process. We calculate that

$$
\begin{aligned}
& \sup _{Y \in \mathcal{A}(y)} \mathbb{E}\left[-\exp \left(-A \limsup _{T \rightarrow \infty} C_{T}^{Y}\right)\right] \\
\geq & \mathbb{E}\left[-\exp \left(-A \limsup _{T \rightarrow \infty} C_{T}^{Y^{p}}\right)\right] \\
\geq & \mathbb{E}\left[-\exp \left(-A C_{T_{p}+\tau_{p}}^{Y^{p}}\right)\right] \\
= & -\exp \left(-A \widetilde{C}+A \int_{0}^{T_{p}} \xi_{t+\tau_{p}}^{p} F\left(\xi_{t+\tau_{p}}^{p}\right) d t\right) \mathbb{E}\left[\exp \left(-\int_{0}^{T_{p}+\tau_{p}} A Y_{t}^{p} d W_{t}\right)\right] \\
= & -\exp \left(-A \widetilde{C}+A \int_{0}^{T_{p}} \xi_{t+\tau_{p}}^{p} F\left(\xi_{t+\tau_{p}}^{p}\right) d t\right) \mathbb{E}\left[\exp \left(-A y W_{\tau_{p}}-\int_{\tau_{p}}^{T_{p}+\tau_{p}} A Y_{t}^{p} d W_{t}\right)\right] \\
= & -\exp \left(-A y p-A \widetilde{C}+A \int_{0}^{T_{p}} \xi_{t+\tau_{p}}^{p} F\left(\xi_{t+\tau_{p}}^{p}\right) d t\right) \mathbb{E}\left[\exp \left(\int_{\tau_{p}}^{T_{p}+\tau_{p}} \frac{1}{2} A^{2}\left(Y_{t}^{p}\right)^{2} d t\right)\right] \\
= & -\exp \left(-A y p-A \widetilde{C}+A \int_{0}^{T_{p}} \xi_{t+\tau_{p}}^{p} F\left(\xi_{t+\tau_{p}}^{p}\right) d t+\int_{0}^{T_{p}} \frac{1}{2} A^{2}\left(Y_{t+\tau_{p}}^{p}\right)^{2} d t\right),
\end{aligned}
$$

where $\widetilde{C}=c+s y-\frac{1}{2} \alpha y^{2}$, and notice that the two integrals in the above line are two constants. Taking $p$ to $+\infty$ gives

$$
\lim _{p \rightarrow \infty} \mathbb{E}\left[-\exp \left(-A C_{T_{p}+\tau_{p}}^{Y^{p}}\right)\right]=0
$$


and hence we conclude that the associated value function is degenerate. Moreover, Jensen's inequality results in

$$
\lim _{p \rightarrow \infty}-\exp \left(-A \mathbb{E}\left[C_{T_{p}+\tau_{p}}^{Y_{p}^{p}}\right]\right) \geq \lim _{p \rightarrow \infty} \mathbb{E}\left[-\exp \left(-A C_{T_{p}+\tau_{p}}^{Y^{p}}\right)\right]=0,
$$

which implies that

$$
\lim _{p \rightarrow \infty} \mathbb{E}\left[C_{T_{p}+\tau_{p}}^{Y^{p}}\right]=\infty
$$

However, $Y^{p}$ clearly violates (2.3) and (2.4). This shows that (2.3) and (2.4) are not only convenient from a mathematical point of view, but also necessary in order for the problem to be well formulated.

\section{Problem simplification}

The main purpose of this section is to reduce problem $(2.32)$ to a deterministic optimization problem. Set $\bar{\delta}_{A}=-\bar{\delta} / A$, where $\bar{\delta}$ is the negative number appearing in $(2.2)$ and $A$ is the risk aversion parameter appearing in the agent's utility function $U$. We make the following further assumptions.

Assumption 3.1. The agent's initial stock position $y$ is strictly less than $\bar{\delta}_{A}$.

Assumption 3.2. The drift $\mu$ of the Lévy process $L$ satisfies $\mu \leq 0$.

Assumption 3.1 restricts the size of the agent's initial position in order to ensure that the objective function is finite and well defined. If we do not impose this restriction, then the market risk associated with the position is so large that the agent would want to reduce the position immediately at any finite cost, which is not possible with an absolutely continuous strategy. Assumption 3.2 excludes a degenerate case of our reduced problem (see the discussion after equation (3.28) $)$.

Define a function $\kappa_{A}:\left[0, \bar{\delta}_{A}\right) \rightarrow \mathbb{R}$ by $\kappa_{A}(x)=\kappa(-A x)$, where $\kappa$ is the cumulant generating function of $L_{1}$, that is

$$
\kappa(x)=\ln \left(\mathbb{E}\left[\mathrm{e}^{x L_{1}}\right]\right), \quad x \in \mathbb{R} .
$$

This function will play an important role in the sequel.

Lemma 3.3. The function $\kappa_{A}$ has the following properties

(i) $\kappa_{A}(0)=0$,

(ii) $\kappa_{A}$ is strictly convex,

(iii) if $\mu=0$, then $\lim _{x \rightarrow 0} \frac{\kappa_{A}(x)}{x^{2}}=K$, for some constant $K>0$, 
(iv) if $\mu \neq 0$, then $\lim _{x \rightarrow 0} \frac{\kappa_{A}(x)}{x}=-A \mu$.

Proof. Let $\psi(u)$ be given by the Lévy-Khintchine representation of $L$. Then for all $u \in\left[0, \bar{\delta}_{A}\right)$, we have

$$
\kappa_{A}(u)=\psi(\mathrm{i} A u)=-A \mu u+\frac{1}{2} A^{2} u^{2} \sigma^{2}+\int_{\mathbb{R}}\left(\mathrm{e}^{-A u x}-1+A u x\right) \nu(d x) .
$$

Therefore, $\kappa_{A}(0)=0$ follows directly.

For part (ii), observe that $-A \mu u, \frac{1}{2} A^{2} u^{2} \sigma^{2}$ and $\mathrm{e}^{-A u x}-1+A u x$ are all convex in $u$, and in particular that $\frac{1}{2} A^{2} u^{2} \sigma^{2}$ and $\mathrm{e}^{-A u x}-1+A u x$ are strictly convex in $u$. Thus, with reference to (3.2), the strict convexity of $\kappa_{A}$ can be concluded from the assumption that $L$ is non-trivial.

Next we prove part (iii). Let $\mu=0$. In view of $(3.2)$, in order to proof $\lim _{x \rightarrow 0} \frac{\kappa_{A}(x)}{x^{2}}=$ $K>0$, it suffices to show that

$$
\lim _{u \rightarrow 0} \int_{\mathbb{R}}\left(\frac{e^{-A u x}-1+A u x}{A^{2} u^{2}}\right) \nu(d x)=K^{\prime}
$$

for some constant $K^{\prime}>0$. Let $0<A \bar{u}<\bar{\delta}_{A}$. It can be checked that for all $u \in(0, \bar{u})$,

$$
\left|\frac{e^{-A u x}-1+A u x}{A^{2} u^{2}}\right|<\frac{x^{2}}{2}, \quad \text { if } x>0,
$$

and

$$
\left|\frac{e^{-A u x}-1+A u x}{A^{2} u^{2}}\right|<\frac{e^{-A \bar{u} x}-1+A \bar{u} x}{A^{2} \bar{u}^{2}}, \quad \text { if } x<0 .
$$

Because of the finite second moment of $L_{1}$ and the fact that $\kappa_{A}(\bar{u})<\infty$, both $\frac{x^{2}}{2}$ and $\frac{e^{-A \bar{u} x}-1+A \bar{u} x}{A^{2} \bar{u}^{2}}$ are $\nu$-integrable. Thus, by the dominated convergence theorem, it follows that

$$
\lim _{u \rightarrow 0} \int_{\mathbb{R}}\left(\frac{e^{-A u x}-1+A u x}{A^{2} u^{2}}\right) \nu(d x)=\int_{\mathbb{R}} \frac{x^{2}}{2} \nu(d x)=K^{\prime}
$$

where $K^{\prime}$ is some strictly positive constant.

Finally we prove part (iv). Let $\mu \neq 0$. Then $\lim _{x \rightarrow 0} \frac{\kappa_{A}(x)}{x}=-A \mu$ follows from $(3.2$ as well as part (iii).

Lemma 3.4. Let $Y$ be a continuous process starting form $y \in\left[0, \bar{\delta}_{A}\right)$. Then

$$
\int_{0}^{\infty}\left\|Y_{t}\right\|_{L^{\infty}(\mathbb{P})}^{i} d t<\infty
$$

if and only if

$$
\int_{0}^{\infty} \kappa_{A}\left(\left\|Y_{u}\right\|_{L^{\infty}(\mathbb{P})}\right) d u<\infty
$$


where $i=1$ if $\mu<0$, and $i=2$ if $\mu=0$. Moreover, with $\mu>0$,

$$
\int_{0}^{\infty}\left\|Y_{t}\right\|_{L^{\infty}(\mathbb{P})} d t<\infty
$$

implies

$$
\int_{0}^{\infty} \kappa_{A}\left(\left\|Y_{u}\right\|_{L^{\infty}(\mathbb{P})}\right) d u<\infty
$$

Proof. Let $\mu=0$. Then Lemma 3.3 (iii) implies that there exist strictly positive constants $\bar{x}$, $C_{1}$ and $C_{2}$ such that $C_{1} x^{2}<\kappa_{A}(x)<C_{2} x^{2}$, for all $x \in(0, \bar{x})$. Suppose that $\int_{0}^{\infty}\left\|Y_{t}\right\|_{L^{\infty}(\mathbb{P})}^{2} d t<$ $\infty$. Then $Y_{t}$ tends to zero as $t$ tends to infinity. Hence, there exist $s>0$, such that $\left\|Y_{t}\right\|_{L^{\infty}(\mathbb{P})} \in$ $(0, \bar{x})$, for all $t>s$. Then

$$
C_{1} \int_{s}^{\infty}\left\|Y_{t}\right\|_{L^{\infty}(\mathbb{P})}^{2} d t<\int_{s}^{\infty} \kappa_{A}\left(\left\|Y_{t}\right\|_{L^{\infty}(\mathbb{P})}\right) d t<C_{2} \int_{s}^{\infty}\left\|Y_{t}\right\|_{L^{\infty}(\mathbb{P})}^{2} d t,
$$

from which it follows that $\int_{s}^{\infty} \kappa_{A}\left(\left\|Y_{u}\right\|_{L^{\infty}(\mathbb{P})}\right) d u<\infty$. Since $\left\|Y_{t}\right\|_{L^{\infty}(\mathbb{P})}$ is bounded for $t \in$ $[0, s]$, we have $\int_{0}^{s} \kappa_{A}\left(\left\|Y_{u}\right\|_{L^{\infty}(\mathbb{P})}\right) d u<\infty$. A similar argument together with the inequality (3.11) also establish the reverse implication. The proofs regarding the cases of $\mu<0$ and $\mu>0$ are similar to above.

In order to reduce problem (2.32), we also require the following technical result.

Lemma 3.5. For any $Y \in \mathcal{A}(y)$, the process $M^{Y}$ given by

$$
M_{t}^{Y}=\exp \left(\int_{0}^{t}-A Y_{u-} d L_{u}-\int_{0}^{t} \kappa_{A}\left(Y_{u}\right) d u\right), \quad t \geq 0,
$$

is a uniformly integrable martingale.

Proof. By Itô's formula and using the expression of $\kappa_{A}$ in 3.2 we calculate that

$$
\begin{aligned}
M_{t}^{Y}=1- & \int_{0}^{t} M_{u-}^{Y} A Y_{u-}\left(\left(\mu-\int_{\mathbb{R}} x \nu(d x)\right) d u+\sigma d W_{u}\right) \\
& \quad-\int_{0}^{t} M_{u-}^{Y}\left(\kappa_{A}\left(Y_{u-}\right)-\frac{1}{2} A^{2} Y_{u-}^{2} \sigma^{2}\right) d u \\
& \quad+\int_{0}^{t} \int_{\mathbb{R}} M_{u-}^{Y}\left(e^{-A Y_{u-} x}-1\right)((N(d u, d x)-\nu(d x) d u)+\nu(d x) d u) \\
= & 1-\int_{0}^{t} M_{u-}^{Y} A Y_{u-} \sigma d W_{u}+\int_{0}^{t} \int_{\mathbb{R}} M_{u-}\left(e^{-A Y_{u-} x}-1\right)(N(d u, d x)-\nu(d x) d u),
\end{aligned}
$$


which shows that $M$ is a local martingale. Define

$$
X_{t}=\int_{0}^{t}-A Y_{u-} d \tilde{L}_{u} \quad \text { and } \quad K(\theta)_{t}=\int_{0}^{t} \tilde{\kappa}_{A}\left(\theta Y_{u}\right) d u
$$

where $\theta \in[0,1], Y \in \mathcal{A}(y)$ with $y \in\left[0, \bar{\delta}_{A}\right), \tilde{L}$ is the martingale part of $L$ and $\tilde{\kappa}_{A}$ is equal to $\kappa_{A}$ with $\mu=0$. It can be checked that the process $M^{Y}$ in 3.12 can be rewritten as

$$
M^{Y}=\exp (X-K(1))
$$

With reference to Definition 3.1 and Theorem 3.2 in Kallsen and Shiryaev $(2002)$, in order to show $M^{Y}$ is a uniformly integrable martingale, it is sufficent to check that

$$
\lim _{\delta \downarrow 0} \sup _{t \in \mathbb{R}_{+}} \delta \log \left(\mathbb{E}\left[\exp \left(\frac{1}{\delta}\left((1-\delta) K(1)_{t}-K(1-\delta)_{t}\right)\right)\right]\right)=0,
$$

for $\delta \in(0,1)$. Observe that

$$
\begin{aligned}
& \lim _{\delta \downarrow 0} \sup _{t \in \mathbb{R}_{+}} \delta \log \left(\mathbb{E}\left[\exp \left(\frac{1}{\delta}\left((1-\delta) K(1)_{t}-K(1-\delta)_{t}\right)\right)\right]\right) \\
\leq & \lim _{\delta \downarrow 0} \sup _{t \in \mathbb{R}_{+}} \delta \log \left(\exp \left(\left\|\frac{1}{\delta}\left((1-\delta) K(1)_{t}-K(1-\delta)_{t}\right)\right\|_{L^{\infty}(\mathbb{P})}\right)\right) \\
= & \lim _{\delta \downarrow 0} \sup _{t \in \mathbb{R}_{+}}\left\|(1-\delta) K(1)_{t}-K(1-\delta)_{t}\right\|_{L^{\infty}(\mathbb{P})} \\
= & \lim _{\delta \downarrow 0} \sup _{t \in \mathbb{R}_{+}}\left\|(1-\delta) \int_{0}^{t} \tilde{\kappa}_{A}\left(Y_{u}\right) d u-\int_{0}^{t} \tilde{\kappa}_{A}\left((1-\delta) Y_{u}\right) d u\right\|_{L^{\infty}(\mathbb{P})} \\
\leq & \lim _{\delta \downarrow 0} \sup _{t \in \mathbb{R}_{+}} \int_{0}^{t}\left\|(1-\delta) \tilde{\kappa}_{A}\left(Y_{u}\right)-\tilde{\kappa}_{A}\left((1-\delta) Y_{u}\right)\right\|_{L^{\infty}(\mathbb{P})} d u \\
\leq & \lim _{\delta \downarrow 0} \int_{0}^{\infty}\left\|(1-\delta) \tilde{\kappa}_{A}\left(Y_{u}\right)-\tilde{\kappa}_{A}\left((1-\delta) Y_{u}\right)\right\|_{L^{\infty}(\mathbb{P})} d u .
\end{aligned}
$$

For $\delta \in(0,1)$, we have that

$$
\begin{aligned}
& \left\|(1-\delta) \tilde{\kappa}_{A}\left(Y_{u}\right)-\tilde{\kappa}_{A}\left((1-\delta) Y_{u}\right)\right\|_{L^{\infty}(\mathbb{P})} \\
\leq & \left\|(1-\delta) \tilde{\kappa}_{A}\left(Y_{u}\right)\right\|_{L^{\infty}(\mathbb{P})}+\left\|\tilde{\kappa}_{A}\left((1-\delta) Y_{u}\right)\right\|_{L^{\infty}(\mathbb{P})} \\
= & (1-\delta) \tilde{\kappa}_{A}\left(\left\|Y_{u}\right\|_{L^{\infty}(\mathbb{P})}\right)+\tilde{\kappa}_{A}\left((1-\delta)\left\|Y_{u}\right\|_{L^{\infty}(\mathbb{P})}\right) \\
\leq & 2 \tilde{\kappa}_{A}\left(\left\|Y_{u}\right\|_{L^{\infty}(\mathbb{P})}\right) .
\end{aligned}
$$

The last two steps are because $\tilde{\kappa}_{A}(x)$ is positive and non-decreasing for $x \geq 0$, which follow from Lemma 3.3 (i), (ii) and (iii). According to (2.3) or 2.4) as well as Lemma 3.4, we have

$$
\int_{0}^{\infty} \tilde{\kappa}_{A}\left(\left\|Y_{t}\right\|_{L^{\infty}(\mathbb{P})}\right) d t<\infty .
$$


Then, by the dominated convergence theorem, 3.17) gives

$$
\begin{aligned}
& \lim _{\delta \downarrow 0} \sup _{t \in \mathbb{R}_{+}} \delta \log \left(\mathbb{E}\left[\exp \left(\frac{1}{\delta}\left((1-\delta) K(1)_{t}-K(1-\delta)_{t}\right)\right)\right]\right) \\
\leq & \int_{0}^{\infty} \lim _{\delta \downarrow 0}\left\|(1-\delta) \tilde{\kappa}_{A}\left(Y_{u}\right)-\tilde{\kappa}_{A}\left((1-\delta) Y_{u}\right)\right\|_{L^{\infty}(\mathbb{P})} d u \\
= & 0 .
\end{aligned}
$$

On the other hand, the convexity of $\tilde{\kappa}_{A}(x)$ and $\tilde{\kappa}_{A}(0)=0$ imply

$$
(1-\delta) \tilde{\kappa}_{A}(x) \geq \tilde{\kappa}_{A}((1-\delta) x), \quad \text { for } \delta \in(0,1),
$$

hence,

$$
(1-\delta) K(1)_{t}-K(1-\delta)_{t} \geq 0 .
$$

Combining this with 3.20 , we get 3.16 .

It follows from Lemma 3.4 and Lemma 3.5 that, for any $Y \in \mathcal{A}(y)$, the process $M^{Y}$ is a strictly positive martingale closed by $M_{\infty}^{Y}$. We can therefore define a new probability measure $\mathbb{Q}^{Y}$ by

$$
\frac{d \mathbb{Q}^{Y}}{d \mathbb{P}}=M_{\infty}^{Y}
$$

Based on the idea in Schied et al. (2010) Theorem 2.8, and with reference to 2.32 and Lemma 3.5, we calculate that

$$
\begin{aligned}
& \inf _{Y \in \mathcal{A}(y)} \mathbb{E}\left[\exp \left(-\int_{0}^{\infty} A Y_{t-} d L_{t}+A \int_{0}^{\infty} \xi_{t} F\left(\xi_{t}\right) d t\right)\right] \\
= & \inf _{Y \in \mathcal{A}(y)} \mathbb{E}\left[\exp \left(-\int_{0}^{\infty} A Y_{t-} d L_{t}-\int_{0}^{\infty} \kappa_{A}\left(Y_{t}\right) d t+\int_{0}^{\infty}\left(\kappa_{A}\left(Y_{t}\right)+A \xi_{t} F\left(\xi_{t}\right)\right) d t\right)\right] \\
= & \inf _{Y \in \mathcal{A}(y)} \mathbb{E}^{\mathbb{Q}^{Y}}\left[\exp \left(\int_{0}^{\infty}\left(\kappa_{A}\left(Y_{t}\right)+A \xi_{t} F\left(\xi_{t}\right)\right) d t\right)\right] \\
\geq & \inf _{Y \in \mathcal{A}(y)} \exp \left(\int_{0}^{\infty}\left(\kappa_{A}\left(\mathbb{E}^{\mathbb{Q}^{Y}}\left[Y_{t}\right]\right)+\mathbb{E}^{\mathbb{Q}^{Y}}\left[A \xi_{t} F\left(\xi_{t}\right)\right]\right) d t\right) \\
= & \inf _{Y \in \mathcal{A}_{D}(y)} \exp \left[\int_{0}^{\infty}\left(\kappa_{A}\left(Y_{t}\right)+A \xi_{t} F\left(\xi_{t}\right)\right) d t\right],
\end{aligned}
$$

by Jensen's inequality. The reverse inequality is obvious since $\mathcal{A}_{D}(y) \subset \mathcal{A}(y)$. Now suppose that $Y^{*}$ is a solution to problem

$$
\inf _{Y \in \mathcal{A}_{D}(y)} \exp \left[\int_{0}^{\infty}\left(\kappa_{A}\left(Y_{t}\right)+A \xi_{t} F\left(\xi_{t}\right)\right) d t\right] .
$$


Then it must also be a solution to problem 2.32). We conclude that it is sufficient to solve the problem

$$
V(y)=\inf _{Y \in \mathcal{A}_{D}(y)} J(Y), \quad y \in\left[0, \bar{\delta}_{A}\right)
$$

where $V$ denotes the value function and $J$ is given by

$$
J(Y)=\int_{0}^{\infty}\left(\kappa_{A}\left(Y_{t}\right)+A \xi_{t} F\left(\xi_{t}\right)\right) d t
$$

If we take $Y \in \mathcal{A}_{D}(y)$ such that $Y_{t}=(t-\sqrt{y})^{2}$, for $t \in[0, \sqrt{y}]$, and $Y_{t}=0$, for $t>\sqrt{y}$, then it can be checked that

$$
J(Y)=\int_{0}^{\sqrt{y}}\left(\kappa_{A}\left((t-\sqrt{y})^{2}\right)+A(2 \sqrt{y}-2 t) F(2 \sqrt{y}-2 t)\right) d t<\infty,
$$

which implies that $V<\infty$. Lemma 3.3 implies $\kappa_{A} \geq 0$, if $\mu \leq 0$. Hence we have $0 \leq V<\infty$, for all $\mu \leq 0$.

Assumption 3.2 excludes some degeneracy. To see this, suppose $\mu>0$. Then Lemma 3.3 (iv) implies that there exists some constant $z>0$ such that $-\infty<\kappa_{A}(z)<0$. Suppose that the agent's initial stock position is $z$ and consider the strategy $Y \in \mathcal{A}_{D}(z)$ satisfying $Y_{t}^{\prime}=-\xi_{t}=0$ for $t \in[0, s]$ with some $s>0$. Then

$$
V(z) \leq \int_{0}^{s} \kappa_{A}(z) d t+V(z)=s \kappa_{A}(z)+V(z) .
$$

This can happen only if $V(z)=-\infty$. Let $\bar{Y} \in \mathcal{A}_{D}(y)$ with $y \geq z$ and set $t_{z}=\inf \{t \geq 0 \mid$ $\left.\bar{Y}_{t}=z\right\}<\infty$. Then

$$
V(y) \leq \int_{0}^{t_{z}}\left(\kappa_{A}\left(\bar{Y}_{t}\right)+A \bar{\xi}_{t} F\left(\bar{\xi}_{t}\right)\right) d t+V(z)
$$

which implies that $V(y)=-\infty$. As $z$ can be chosen to be arbitrarily close to zero, it follows that $V(y)=-\infty$, for all $y \in\left(0, \bar{\delta}_{A}\right)$. We therefore conclude that the value function is degenerate when $\mu>0$. Let $y \in\left(0, \bar{\delta}_{A}\right)$, and suppose (in order to get a contradiction) that there exists an optimal strategy $Y^{*} \in \mathcal{A}_{D}(y)$. Define $\tilde{\kappa}_{A}$ to be the function which is identical to $\kappa_{A}$ with $\mu=0$. Then with reference to the Lévy-Khintchine representation of $L$ (see (3.2)), we have $\kappa_{A}(x)=-A \mu x+\tilde{\kappa}_{A}(x)$. By Assumption 2.3 and Lemma 3.3, we have that $\widetilde{\kappa}_{A}\left(Y_{t}^{*}\right)+A \xi_{t} F\left(\xi_{t}^{*}\right)$ is positive. Thus,

$$
V(y)=\int_{0}^{\infty}\left(-A \mu Y_{t}^{*}+\tilde{\kappa}_{A}\left(Y_{t}^{*}\right)+A \xi_{t}^{*} F\left(\xi_{t}^{*}\right)\right) d t=-\infty, \quad \mu>0,
$$

implies $\int_{0}^{\infty} Y_{t}^{*} d t=\infty$, which contradicts the definition of an admissible strategy. We conclude that if $\mu>0$, then no optimal admissible liquidation strategy exists. 
Remark 3.6. It is mentioned in Schied et al. (2010) that for the Almgren-Chriss model with Brownian motion describing the unaffected stock price, the problem of optimizing the final cost/reward for an agent with CARA preferences over a set of adapted strategies provides the same optimal solution as for the problem of optimizing for the same model over deterministic strategies, but with a mean-variance optimization criterion. When the unaffected stock price is not a Brownian motion, but a general Lévy process, this relationship no longer holds. To see this, we know that for our optimization problem, the set of admissible strategies $\mathcal{A}(y)$ can be replaced by $\mathcal{A}_{D}(y)$. Then in view of $(2.30)$, it is sufficient to consider

$$
\inf _{Y \in \mathcal{A}_{D}(y)} \mathbb{E}\left[\mathrm{e}^{-A C_{\infty}^{Y}}\right]
$$

where

$$
C_{\infty}^{Y}=c+s y-\frac{1}{2} \alpha y^{2}+\int_{0}^{\infty} Y_{t-} d L_{t}-\int_{0}^{\infty} \xi_{t} F\left(\xi_{t}\right) d t
$$

It can be calculated that

$$
\mathbb{E}\left[C_{\infty}^{Y}\right]=c+s y-\frac{1}{2} \alpha y^{2}+\mu \int_{0}^{\infty} Y_{t} d t-\int_{0}^{\infty} \xi_{t} F\left(\xi_{t}\right) d t
$$

and

$$
\operatorname{Var}\left(C_{\infty}^{Y}\right)=\sigma^{2} \int_{0}^{\infty} Y_{t}^{2} d t+\int_{0}^{\infty}\left(\int_{\mathbb{R}} Y_{t}^{2} x^{2} \nu(d x)\right) d t
$$

Then,

$$
\begin{aligned}
& \mathbb{E}\left[\exp \left(-A C_{\infty}^{Y}\right)\right] \\
= & \exp \left[-A \mathbb{E}\left[C_{\infty}^{Y}\right]+\frac{1}{2} A^{2} \sigma^{2} \int_{0}^{\infty} Y_{t}^{2} d t+\int_{0}^{\infty} \int_{\mathbb{R}}\left(e^{-A Y_{t} x}-1+A Y_{t} x\right) \nu(d x) d t\right] \\
= & \exp \left[-A \mathbb{E}\left[C_{\infty}^{Y}\right]+\frac{1}{2} A^{2} \operatorname{Var}\left(C_{\infty}^{Y}\right)+\int_{0}^{\infty} \int_{\mathbb{R}}\left(e^{-A Y_{t} x}-1+A Y_{t} x-\frac{1}{2} A^{2} Y_{t}^{2} x^{2}\right) \nu(d x) d t\right] .
\end{aligned}
$$

From the above expression, it is clear that the problem is equivalent to

$$
\sup _{Y \in \mathcal{A}_{D}(y)} \mathbb{E}\left[C_{\infty}^{Y}\right]-\frac{1}{2} A \operatorname{Var}\left(C_{\infty}^{Y}\right)
$$

if $\nu(\mathbb{R}) \equiv 0$, i.e. if the Lévy process $L$ has no jumps. However, for a general Lévy process this equivalence does not hold.

Remark 3.7. Suppose that the agent is also allowed to buy shares. Then in order for the final cash position to be well-defined, we need, in addition to the conditions in Definition 2.2. to assume that any admissible strategy $Y$ satisfy $\lim _{t \rightarrow \infty} t\left\|Y_{t}\right\|_{L^{\infty}(\mathbb{P})}=0$ (see Lemma 2.5 and 
proof of Proposition 2.6 for more details). We also suppose $Y$ is non-negative, that $Y_{t}<\bar{\delta}_{A}$ for all $t \geq 0$, and that $Y_{t}=y+\int_{0}^{t} \xi_{u} d u$ with $\xi_{t} \in \mathbb{R}$. Denote by $\mathcal{A}^{ \pm}(y)$ the set of all such admissible strategies, and by $\mathcal{A}_{D}^{ \pm}(y)$ the collection of all deterministic admissible strategies. Then by similar arguments as previously, the liquidation problem can be reduced to

$$
V(y)=\inf _{Y \in \mathcal{A}_{D}^{ \pm}(y)} \int_{0}^{\infty}\left(\kappa_{A}\left(Y_{t}\right)+A\left|\xi_{t}\right| F\left(\left|\xi_{t}\right|\right)\right) d t .
$$

Let $Y \in \mathcal{A}_{D}^{ \pm}(y)$ be a strategy that involves intermediate buying. Then there exist times $r$ and $s$ with $r<s$ such that $Y_{r}=Y_{s}$ and $Y_{t}>Y_{r}$ for all $t \in(r, s)$. Consider an admissible strategy $X$ such that $X_{t}=Y_{r}$ for $t \in(r, s)$ and $X_{t}=Y_{t}$ for $t \in[0, r] \cup[s, \infty)$. Then with reference to Lemma 3.3 ,

$$
\int_{r}^{s}\left(\kappa_{A}\left(X_{u}\right)+A\left|\xi_{u}^{X}\right| F\left(\left|\xi_{u}^{X}\right|\right)\right) d u=\kappa_{A}\left(X_{r}\right)(s-r)<\int_{r}^{s}\left(\kappa_{A}\left(Y_{u}\right)+A\left|\xi_{u}\right| F\left(\left|\xi_{u}\right|\right)\right) d u
$$

where $\xi^{X}$ is the speed process associated with $X$. Therefore, $J(X)<J(Y)$. This shows $Y$ is not optimal. So even if it is allowed, it is not optimal to do any intermediate buying of shares.

\section{Solution to the problem}

With reference to the previous section, recall that the original optimal liquidation problem 2.29 is equivalent to solving

$$
V(y)=\inf _{Y \in \mathcal{A}_{D}(y)} \int_{0}^{\infty}\left(\kappa_{A}\left(Y_{t}\right)+A \xi_{t} F\left(\xi_{t}\right)\right) d t
$$

with

$$
d Y_{t}=-\xi_{t} d t, \quad Y_{0}=y \in\left[0, \bar{\delta}_{A}\right)
$$

According to the theory of optimal control, the corresponding Hamilton-Jacobi-Bellman equation is given by

$$
\kappa_{A}(y)+\inf _{x \geq 0}\left\{A x F(x)-x v^{\prime}(y)\right\}=0
$$

with associated boundary condition $v(0)=0$. Let $G:[0, \infty) \rightarrow[0, \infty)$ denote the inverse function of $x \mapsto x^{2} F^{\prime}(x)$. Assumption 2.3 and Lemma 2.4 together imply that $G$ is a continuous, strictly increasing function satisfying $G(0)=0$. The next lemma states an additional property of the function $G$ that is used in the proofs of Proposition 4.2 and Theorem 4.3 .

Lemma 4.1. The function $x \mapsto \frac{x}{G(x)}$ is continuous on $[0, \infty)$. 
Proof. Assumption 2.3 and Lemma 2.4 imply that $G$ is continuous and $G(0)=0$. Therefore, it is sufficient to check that $\lim _{x \rightarrow 0} \frac{x}{G(x)}<\infty$. Let $x=u^{2} F^{\prime}(u)$. Then it follows that $\frac{x}{G(x)}=$ $u F^{\prime}(u)$. Hence, the result follows from the fact that $u \rightarrow 0$, as $x \rightarrow 0$, and $\lim _{u \rightarrow 0} u F^{\prime}(u)=0$ (see Lemma 2.4).

Proposition 4.2. Equation (4.3) with boundary condition $v(0)=0$ has a classical solution given by

$$
v(y)=\int_{0}^{y}\left\{\frac{\kappa_{A}(u)}{G\left(\frac{\kappa_{A}(u)}{A}\right)}+A F\left(G\left(\frac{\kappa_{A}(u)}{A}\right)\right)\right\} d u, \quad 0 \leq y<\bar{\delta}_{A} .
$$

Proof. We first show that the function $v$ given by (4.4) is continuously differentiable, and note that it is sufficient to show that $v^{\prime}(y)=\frac{\kappa_{A}(y)}{G\left(\frac{\kappa_{A}(y)}{A}\right)}+A F\left(G\left(\frac{\kappa_{A}(y)}{A}\right)\right)$ is continuous on $\left[0, \bar{\delta}_{A}\right)$. This is the case if $x \mapsto \frac{x}{G(x)}$ is continuous for $x \geq 0$. But this is demonstrated by Lemma 4.1 .

Recall that the Hamilton-Jacobi-Bellman equation in our problem is

$$
\kappa_{A}(y)+\inf _{x \geq 0}\left\{A x F(x)-x v^{\prime}(y)\right\}=0 .
$$

In order to prove that $v$ in (4.4) is a solution to this equation, because $A x F(x)-x v^{\prime}(y)$ is strictly convex in $x$, it is enough to show that for all $y \in\left[0, \bar{\delta}_{A}\right)$, there exists $x^{*} \geq 0$ such that

$$
A x^{*} F^{\prime}\left(x^{*}\right)+A F\left(x^{*}\right)-v^{\prime}(y)=0
$$

and

$$
\kappa_{A}(y)+A x^{*} F\left(x^{*}\right)-x^{*} v^{\prime}(y)=0,
$$

where the equality in 4.6 comes from the first-order condition of optimality of the expression $A x F(x)-x v^{\prime}(y)$. But with $v^{\prime}(y)=\frac{\kappa_{A}(y)}{G\left(\frac{\kappa_{A}(y)}{A}\right)}+A F\left(G\left(\frac{\kappa_{A}(y)}{A}\right)\right)$, it can be checked that $x^{*}=$ $G\left(\frac{\kappa_{A}(y)}{A}\right)$ satisfies both 4.6 and 4.7 . The boundary condition $v(0)=0$ is a consequence of the expression of $v(y)$ and the continuity of $v(y)$ at $y=0$.

The next result provides an expression for the optimal liquidation strategy, and states that the value function $V$ identifies with the function $v$ in (4.4).

Theorem 4.3. Let $y \in\left[0, \bar{\delta}_{A}\right)$. Define

$$
\tau=\int_{0}^{y} \frac{1}{G\left(\frac{\kappa_{A}(u)}{A}\right)} d u
$$

Let $Y^{*}$ satisfy

$$
\int_{Y_{t}^{*}}^{y} \frac{1}{G\left(\frac{\kappa_{A}(u)}{A}\right)} d u=t, \quad \text { if } t \leq \tau, \quad \text { and } \quad Y_{t}^{*}=0, \quad \text { if } t>\tau .
$$


Then $Y^{*} \in \mathcal{A}_{D}(y)$, and its associated speed process $\xi^{*}$ satisfies

$$
\xi_{t}^{*}=G\left(\frac{\kappa_{A}\left(Y_{t}^{*}\right)}{A}\right), \quad \text { for all } t \geq 0 .
$$

Moreover, $V$ in (3.26) is equal to $v$ in (4.4), for all $y \in\left[0, \bar{\delta}_{A}\right)$, and $Y^{*}$ is the unique optimal liquidation strategy for problem (2.29).

Proof. We know that when $t \leq \tau$,

$$
\int_{Y_{t}^{*}}^{y} \frac{1}{G\left(\frac{\kappa_{A}(u)}{A}\right)} d u=t
$$

from which it follows that

$$
\xi_{t}^{*}=-\frac{d Y_{t}^{*}}{d t}=G\left(\frac{\kappa_{A}\left(Y_{t}^{*}\right)}{A}\right), \quad t \leq \tau
$$

On the other hand, when $t>\tau, Y_{t}^{*}=0$. Hence,

$$
\xi_{t}^{*}=0=G\left(\frac{\kappa_{A}\left(Y_{t}^{*}\right)}{A}\right), \quad t>\tau
$$

We next prove that $Y^{*} \in \mathcal{A}_{D}(y)$. It is clear that $Y^{*}$ is deterministic and absolutely continuous. The non-negativity of $G$ implies that $Y^{*}$ is non-increasing. It remains to show that if $\mu<0$, then $\int_{0}^{\infty} Y_{t}^{*} d t<\infty$; and if $\mu=0$, then $\int_{0}^{\infty}\left(Y_{t}^{*}\right)^{2} d t<\infty$. However, with reference to Lemma 3.4 , it is enough to check that

$$
\int_{0}^{\infty} \kappa_{A}\left(Y_{t}^{*}\right) d t=\int_{0}^{\tau} \kappa_{A}\left(Y_{t}^{*}\right) d t<\infty
$$

By a change of variable, we have that

$$
\int_{0}^{\tau} \kappa_{A}\left(Y_{t}^{*}\right) d t=\int_{y}^{0}-\frac{\kappa_{A}\left(Y_{t}^{*}\right)}{G\left(\frac{\kappa_{A}\left(Y_{t}^{*}\right)}{A}\right)} d Y_{t}^{*}<\infty
$$

where the finiteness is due to the continuity of the integrand on the compact interval $[0, y]$, which is implied by Lemma 4.1 .

With reference to (4.6) and (4.7), the function $v$ in (4.4) satisfies

$$
\kappa_{A}(y)+A \xi F(\xi)-\xi v^{\prime}(y) \geq 0, \quad \text { for all } \xi \geq 0,
$$

and equality holds only when $\xi=G\left(\frac{\kappa_{A}(y)}{A}\right)$. Let $Y \in \mathcal{A}_{D}(y)$. Observe that

$$
v\left(Y_{T}\right)=v(y)-\int_{0}^{T} v^{\prime}\left(Y_{t}\right) \xi_{t} d t
$$


Taking $T$ to infinity and using the boundary condition $v(0)=0$, it follows that

$$
v(y)=\int_{0}^{\infty} v^{\prime}\left(Y_{t}\right) \xi_{t} d t
$$

Then by (4.16) we have

$$
v(y) \leq \int_{0}^{\infty}\left(\kappa_{A}\left(Y_{t}\right)+A \xi_{t} F\left(\xi_{t}\right)\right) d t
$$

Now consider the strategy $Y^{*}$ in 4.9 , which has a speed process $\xi^{*}$ satisfying $\xi_{t}^{*}=G\left(\frac{\kappa_{A}\left(Y_{t}^{*}\right)}{A}\right)$, for all $t \geq 0$. Then,

$$
\kappa_{A}\left(Y_{t}^{*}\right)+A \xi_{t}^{*} F\left(\xi_{t}^{*}\right)-\xi_{t}^{*} v^{\prime}\left(Y_{t}^{*}\right)=0, \quad t \geq 0
$$

hence,

$$
v(y)=\int_{0}^{\infty}\left(\kappa_{A}\left(Y_{t}^{*}\right)+A \xi_{t} F\left(\xi_{t}^{*}\right)\right) d t .
$$

This together with 4.19 implies that $V(y)=v(y)$, for all $y \in\left[0, \bar{\delta}_{A}\right)$. Therefore, with reference to the analysis after equation (3.24), we get that $Y^{*}$ is the unique optimal strategy to problem 2.29 .

Note that since is $G$ continuous, 4.10) implies that the strategy $Y^{*}$ in $(4.9)$ is continuously differentiable. Since the functions $\kappa_{A}$ and $G$ are both strictly increasing, it follows from 4.10) that with a larger stock position at time $t$, the associated optimal liquidation speed at time $t$ is larger. Moreover, it can be shown by the strict convexity of the cumulant generating function of $L_{1}$ that $A \mapsto \kappa_{A}(x) / A$ is strictly increasing. Hence, the optimal liquidation speed at any time is strictly increasing in the risk aversion parameter $A$. These two relations coincide with the intuition that with a larger position of shares, the agent potentially encounters bigger risk from the market volatility, as any tiny fluctuation of the stock price will be amplified by the large number of shares held. It is therefore optimal to liquidate faster. Also the more risk averse the agent is, the more the agent cares about the volatility risk, which makes the agent employ a more rapid liquidation strategy. Observe that given an initial stock position $y \in\left[0, \bar{\delta}_{A}\right)$, the quantity $\tau$ in 4.8 indicates the liquidation time for the optimal liquidation strategy $Y^{*}$. Depending on the properties of the temporary impact function $F, \tau$ may or may not be finite. The next result provides some sufficient conditions for the optimal liquidation strategy $Y^{*}$ to have a finite liquidation time.

Proposition 4.4. Assume that $y>0$.

(i) If $\mu<0$ and there exist constants $p<1$ and $K>0$ such that $\lim _{x \rightarrow 0} x^{p} F^{\prime}(x)=K$, then it holds that $\tau<\infty$. 
(ii) If $\mu=0$ and there exist constants $p<1$ and $K>0$ such that $\lim _{x \rightarrow 0} x^{p} F^{\prime}(x)=K$, then $\tau=\infty$ if $p \in[0,1)$ and $\tau<\infty$ if $p<0$.

Proof. Suppose $\mu<0$ and let $p<1$ be such that $\lim _{x \rightarrow 0} x^{p} F^{\prime}(x)=K$, with $K$ being some strictly positive constant. Write $u=x^{2} F^{\prime}(x)$. Then we have

$$
\frac{u^{\frac{1}{2-p}}}{G(u)}=\left(x^{p} F^{\prime}(x)\right)^{\frac{1}{2-p}} \text {. }
$$

By letting $x$ tend to 0 , so $u$ tends to 0 as well, it follows that

$$
\lim _{u \rightarrow 0} \frac{u^{\frac{1}{2-p}}}{G(u)}=K^{\frac{1}{2-p}}
$$

Lemma 3.3 (iv) together with 4.23 gives

$$
\lim _{x \rightarrow 0} \frac{x^{\frac{1}{2-p}}}{G\left(\frac{\kappa_{A}(x)}{A}\right)}=K^{\prime},
$$

for some other constant $K^{\prime}>0$. Therefore, there exist strictly positive constants $K_{1}, K_{2}$ and $\bar{x}$ such that for all $x \in(0, \bar{x})$,

$$
\frac{K_{1}}{x^{\frac{1}{2-p}}}<\frac{1}{G\left(\frac{\kappa_{A}(x)}{A}\right)}<\frac{K_{2}}{x^{\frac{1}{2-p}}} .
$$

Integrating and taking limit on each term gives

$$
\lim _{x \rightarrow 0} \int_{x}^{\bar{x}} \frac{K_{1}}{u^{\frac{1}{2-p}}} d u \leq \lim _{x \rightarrow 0} \int_{x}^{\bar{x}} \frac{1}{G\left(\frac{\kappa_{A}(u)}{A}\right)} d u \leq \lim _{x \rightarrow 0} \int_{x}^{\bar{x}} \frac{K_{2}}{u^{\frac{1}{2-p}}} d u .
$$

Observe that $p<1$ implies $\frac{1}{2-p}<1$, and therefore $\int_{0}^{\bar{x}} \frac{1}{u^{\frac{1}{2-p}}} d u<\infty$. Hence,

$$
\lim _{x \rightarrow 0} \int_{x}^{\bar{x}} \frac{1}{G\left(\frac{\kappa_{A}(u)}{A}\right)} d u<\infty .
$$

Then the required result follows from 4.8 and the fact that $\int_{\bar{x}}^{y} \frac{1}{G\left(\frac{\kappa_{A}(u)}{A}\right)} d u<\infty$, if the initial stock position $y>\bar{x}$.

Next we prove part (ii). Suppose $\mu=0$. Observe that 4.23 implies

$$
\lim _{x \rightarrow 0} \frac{x^{\frac{2}{2-p}}}{G\left(x^{2}\right)}=C
$$


for some constant $C>0$. Combining this with Lemma 3.3 (iii), we obtain

$$
\lim _{x \rightarrow 0} \frac{x^{\frac{2}{2-p}}}{G\left(\frac{\kappa_{A}(x)}{A}\right)}=C^{\prime}
$$

for some other constant $C^{\prime}>0$. Then there exist strictly positive constants $C_{1}, C_{2}$ and $\bar{x}$ such that for all $x \in(0, \bar{x})$,

$$
\frac{C_{1}}{x^{\frac{2}{2-p}}}<\frac{1}{G\left(\frac{\kappa_{A}(x)}{A}\right)}<\frac{C_{2}}{x^{\frac{2}{2-p}}}
$$

Therefore,

$$
\lim _{x \rightarrow 0} \int_{x}^{\bar{x}} \frac{C_{1}}{u^{\frac{2}{2-p}}} d u \leq \lim _{x \rightarrow 0} \int_{x}^{\bar{x}} \frac{1}{G\left(\frac{\kappa_{A}(u)}{A}\right)} d u \leq \lim _{x \rightarrow 0} \int_{x}^{\bar{x}} \frac{C_{2}}{u^{\frac{2}{2-p}}} d u .
$$

If $p<0$, then $\frac{2}{2-p}<1$. Hence $\tau<\infty$ is obtained by the same argument as in (i) of this proof. If $p \in[0,1)$, then $\frac{2}{2-p} \geq 1$. It follows that $\int_{0}^{\bar{x}} \frac{1}{G\left(\frac{\kappa_{A}(u)}{A}\right)} d u=\infty$, and therefore $\tau=\infty$.

\section{Approximation for exponential Lévy model}

In models for stock prices involving Lévy processes, it is common to consider exponential Lévy processes (Madan and Seneta, 1990), (Eberlein and Keller, 1995), (Barndorff-Nielsen, 1997). However, it is common in the optimal liquidation literature to use linear models as opposed to exponential models due to tractability and the short time horizons involved. For practical implementation of our model one could of course directly fit the data to a linear Lévy model. However families of distributions that fit observed stock market data well for the exponential Lévy model are known and obviously the distribution of the jumps change when you take the exponential. We therefore investigate how to linearize exponential Lévy models and how this affects the Lévy measure. To this end, we are going to derive a Lévy process which can be regarded as a linear approximation for the corresponding exponential Lévy process. We show that this Lévy process satisfies all of the assumptions of being the driving process of the unaffected stock price in the liquidation model introduced in previous sections. Therefore, our optimal liquidation strategy derived in the previous section can be regarded as an approximation for the result of the corresponding exponential Lévy model. This linear approximation argument is reasonable since (the majority of) liquidation usually is completed in a very short time.

Consider a non-trivial, one-dimensional, $\mathbb{F}$-adapted Lévy process $\tilde{L}$ which admits the canonical decomposition

$$
\tilde{L}_{t}=\tilde{\mu} t+\tilde{\sigma} \tilde{W}_{t}+\int_{|z| \geq 1} z \tilde{N}(t, d z)+\int_{|z|<1} z(\tilde{N}(t, d z)-t \tilde{\nu}(d z)), \quad t \geq 0
$$


where $\tilde{\mu} \in \mathbb{R}$ and $\tilde{\sigma} \geq 0$ are two constants, $\tilde{W}$ is a standard Brownian motion, $\tilde{N}$ is a Poisson random measure which is independent of $\tilde{W}$ with compensator $t \tilde{\nu}(d z)$, and $\tilde{\nu}$ is the Lévy measure associated with $\tilde{L}$. We assume that $\tilde{L}$ possess the following properties.

Assumption 5.1. We assume that $\tilde{\nu}$ is absolutely continuous with respect to Lebesgue measure, and that

$$
\int_{|z| \geq 1} \mathrm{e}^{2 z} \tilde{\nu}(d z)<\infty
$$

Suppose the unaffected stock price is described by the process $\tilde{S}^{u}$ satisfying

$$
\tilde{S}_{t}^{u}=\tilde{s} \exp \left(\tilde{L}_{t}\right), \quad t \geq 0,
$$

where $\tilde{s}>0$ is some constant denoting the initial stock price. Note that $(5.2)$ ensures $\tilde{S}_{t}^{u}$ is square integrable, for all $t \geq 0$ (by Theorem 3.6 in Kyprianou (2006)). Suppose the affected stock price at time $t \geq 0$ is given by

$$
\tilde{S}_{t}=\tilde{s} \exp \left(\tilde{L}_{t}\right)+I_{t}
$$

where $I_{t}=\alpha\left(Y_{t}-Y_{0}\right)-F\left(\xi_{t}\right)$ is the price impact at time $t$ appearing in the previous liquidation model with function $F$ satisfying Assumption 2.3 (Gatheral and Schied (2011) study a liquidation model of this form where the unaffected stock price is a geometric Brownian motion). By Itô's formula, for all $t \geq 0, \tilde{S}_{t}$ can be rewritten as

$$
\tilde{S}_{t}=\tilde{s}+\int_{0}^{t} \tilde{S}_{u-}^{u} \tilde{m} d u+\int_{0}^{t} \tilde{S}_{u-}^{u} \tilde{\sigma} d \tilde{W}_{u}+\int_{0}^{t} \int_{\mathbb{R}} \tilde{S}_{u-}^{u}\left(\mathrm{e}^{z}-1\right)(\tilde{N}(t, d z)-t \tilde{\nu}(d z))+I_{t}
$$

where $\tilde{m}=\tilde{\mu}+\frac{\tilde{\sigma}^{2}}{2}+\int_{\mathbb{R}}\left(\mathrm{e}^{z}-1-z \mathbb{1}_{\{|z|<1\}}\right) \tilde{\nu}(d z)$. In order to approximate the exponential Lévy model, consider the process $\hat{S}$ such that

$$
\hat{S}_{t}=\tilde{s}+\tilde{s} \tilde{m} t+\tilde{s} \tilde{\sigma} \tilde{W}_{t}+\int_{\mathbb{R}} \tilde{s}\left(\mathrm{e}^{z}-1\right)(\tilde{N}(t, d z)-t \tilde{\nu}(d z))+I_{t}, \quad t \geq 0
$$

which can be considered as a linear approximation of $\tilde{S}$. Recall that the affected stock price in the preceding model is given by

$$
S_{t}=s+L_{t}+I_{t}, \quad t \geq 0,
$$

where $L_{t}=\mu t+\sigma W_{t}+\int_{\mathbb{R}} x(N(t, d x)-t \nu(d x))$. Comparing this to the expression of $\hat{S}_{t}$, it can be seen that if we take $s=\tilde{s}$ and choose $L$ to be such that

$$
L_{t}=\tilde{s} \tilde{m} t+\tilde{s} \tilde{\sigma} \tilde{W}_{t}+\int_{\mathbb{R}} \tilde{s}\left(\mathrm{e}^{z}-1\right)(\tilde{N}(t, d z)-t \tilde{\nu}(d z)), \quad t \geq 0
$$


then it follows that

$$
\hat{S}_{t}=\tilde{s}+L_{t}+I_{t}, \quad \text { for all } t \geq 0 .
$$

We may therefore consider $\hat{S}$ as the affected stock price process in the liquidation model introduced in previous sections. The next proposition verifies that $L$ with the above expression is a Lévy process satisfying Assumption 2.1.

Proposition 5.2. Let $L$ be given by (5.8) and set $\hat{L}=L / \tilde{s}$. Then $\hat{L}$ is an $\mathbb{F}$-adapted Lévy process whose Lévy measure, denoted by $\hat{\nu}$, satisfies

$$
\hat{\nu}(d x)=\frac{1}{x+1} \tilde{f}(\ln (x+1)) d x, \quad x>-1, x \neq 0 .
$$

Therefore, $L$ is an $\mathbb{F}$-adapted Lévy process satisfying Assumption 2.1.

Proof. We show that $\hat{L}$ given by

$$
\hat{L}_{t}=\tilde{m} t+\tilde{\sigma} \tilde{W}_{t}+\int_{0}^{t} \int_{\mathbb{R}}\left(\mathrm{e}^{z}-1\right)(\tilde{N}(d t, d z)-\tilde{\nu}(d z) d t), \quad t \geq 0
$$

is a Lévy process. Define a random measure $\hat{N}: \Omega \times \mathcal{B}([0, \infty)) \otimes \mathcal{B}(\mathbb{R}) \rightarrow \mathbb{Z}_{+}$and a measure $\hat{\nu}: \mathcal{B}(\mathbb{R}) \rightarrow \mathbb{Z}_{+}$to be such that if $B \in \mathcal{B}(\mathbb{R})$ and $B \cap(-1, \infty) \neq \emptyset$, then

$$
\begin{aligned}
\hat{N}(\omega, A, B) & =\tilde{N}(A, \ln (B \cap(-1, \infty)+1))(\omega), \\
\hat{\nu}(B) & =\tilde{\nu}(\ln (B \cap(-1, \infty)+1))
\end{aligned}
$$

otherwise, they are both equal 0 , where $\mathbb{Z}_{+}$is the set of all positive integers and $\ln (B \cap$ $(-1, \infty)+1)=\{\ln (x+1) \mid x \in B \cap(-1, \infty)\}$ ( we have for all $A \in \mathcal{B}([0, \infty))$ and $\omega \in \Omega$, $\tilde{N}(A,\{0\})(\omega)=\tilde{\nu}(\{0\})=0)$. Write $\hat{N}(\cdot, \cdot)=\hat{N}(\omega, \cdot, \cdot)$. Then by writing $x=\mathrm{e}^{z}-1$, it follows from (5.11) that

$$
\hat{L}_{t}=\tilde{m} t+\tilde{\sigma} \tilde{W}_{t}+\int_{0}^{t} \int_{\mathbb{R}} x(\hat{N}(d t, d x)-\hat{\nu}(d x) d t), \quad t \geq 0 .
$$

With reference to Corollary 15.7in Kallenberg (2001), to prove $\hat{L}$ is a Lévy process, it suffices to show that for any $B \in \mathcal{B}(\mathbb{R}),(\hat{N}(t, B))_{t \geq 0}$ is a Poisson process with intensity $\hat{\nu}(B)$ satisfying

$$
\int_{\mathbb{R}}\left(x^{2} \wedge 1\right) \hat{\nu}(d x)<\infty .
$$

But from the definition of $\hat{N}$, it is clear that $(\hat{N}(t, B))_{t \geq 0}$ is a Poisson process. Observe that

$$
\mathbb{E}[\hat{N}(t, B)]=\mathbb{E}[\tilde{N}(t, \ln (B \cap(-1, \infty)+1))]=t \tilde{\nu}(\ln (B \cap(-1, \infty)+1))=t \hat{\nu}(B),
$$


which proves that $\hat{\nu}(B)$ is the intensity of $(\hat{N}(t, B))_{t \geq 0}$. From the Taylor expansion of $\left(\mathrm{e}^{z}-1\right)^{2}$, it can be shown that there exist constants $\bar{z}>0$ and $C>0$ such that for all $z \in(-\bar{z}, \bar{z})$,

$$
\left(\mathrm{e}^{z}-1\right)^{2} \leq C z^{2}
$$

For $\epsilon \in(0,1)$, consider the interval $\mathcal{S}=(\ln (1-\epsilon), \ln (\epsilon+1))$. Then using 5.12 we calculate that for $\epsilon$ close enough to 0 so that $\mathcal{S} \subseteq(-\bar{z}, \bar{z})$, we have

$$
\int_{(-\epsilon, \epsilon)} x^{2} \hat{\nu}(d x)=\int_{\mathcal{S}}\left(\mathrm{e}^{z}-1\right)^{2} \tilde{\nu}(d z) \leq C \int_{\mathcal{S}} z^{2} \tilde{\nu}(d z) \leq C \int_{(-\bar{z}, \bar{z})} z^{2} \tilde{\nu}(d z)<\infty,
$$

where the finiteness follows since $\tilde{\nu}$ is a Lévy measure. Again by (5.12), we obtain

$$
\int_{\mathbb{R} \backslash(-\epsilon, \epsilon)} \hat{\nu}(d x)=\int_{\mathbb{R} \backslash \mathcal{S}} \tilde{\nu}(d z)<\infty,
$$

where the finiteness again follows since $\tilde{\nu}$ is a Lévy measure . This implies that $\hat{\nu}(\mathbb{R} \backslash(-1,1))<$ $\infty$ and $\hat{\nu}((-1,-\epsilon] \cup[\epsilon, 1))<\infty$. Since $x^{2}$ is bounded on $(-1,-\epsilon] \cup[\epsilon, 1)$, together with (5.17), we get

$$
\int_{(-1,1)} x^{2} \hat{\nu}(d x)<\infty
$$

Combining this with $\hat{\nu}(\mathbb{R} \backslash(-1,1))<\infty$, we get 5.14). We therefore conclude that $\hat{N}$ and $\hat{\nu}$ is a Poisson random measure and the Lévy measure associated with the Lévy process $\hat{L}$, respectively. Moreover, we calculate from (5.12) that for $x>-1$ and $x \neq 0$,

$$
\hat{\nu}(d x)=\tilde{\nu}(d(\ln (x+1)))=\tilde{f}(\ln (x+1)) d(\ln (x+1))=\frac{1}{x+1} \tilde{f}(\ln (x+1)) d x .
$$

The relation $L=\tilde{s} \hat{L}$ shows that $L$ is also a Lévy process. The expression of $L$ in 5.8 shows the adaptedness. Next we check that Assumption 2.1 is satisfied by $L$, and note that it is sufficient to check the condition for $\hat{L}$. According to Assumption 5.1, we know $\int_{|z| \geq 1} \mathrm{e}^{2 z} \tilde{\nu}(d z)<\infty$, and since for any $\epsilon>0, \tilde{\nu}(\mathbb{R} \backslash(-\epsilon, \epsilon))<\infty$, it follows that on $[\ln 2, \infty)$, $\mathrm{e}^{2 z}$ and $\mathrm{e}^{z}$ are both $\tilde{\nu}$-integrable and $\tilde{\nu}([\ln 2, \infty))<\infty$. Therefore,

$$
\int_{|x| \geq 1} x^{2} \hat{\nu}(d x)=\int_{[\ln 2, \infty)}\left(\mathrm{e}^{z}-1\right)^{2} \tilde{\nu}(d z)<\infty
$$

which implies that $\hat{L}_{1}$ has finite second moment (by Theorem 3.8 in Kyprianou (2006)). Observe that when $u \leq 0$, we have

$$
\exp \left(u\left(\mathrm{e}^{z}-1\right)\right) \leq 1, \quad \text { for all } z \geq 0 .
$$


Hence,

$$
\int_{|x| \geq 1} \mathrm{e}^{u x} \hat{\nu}(d x)=\int_{[\ln 2, \infty)} \exp \left(u\left(\mathrm{e}^{z}-1\right)\right) \tilde{\nu}(d z)<\infty
$$

from which it follows that $\mathbb{E}\left[\mathrm{e}^{u \hat{L}_{1}}\right]<\infty$, for all $u \leq 0$.

Remark 5.3. From equation (5.23) (in the proof of Proposition 5.2) we know that

$$
\int_{|x| \geq 1} \mathrm{e}^{u x} \hat{\nu}(d x)<\infty, \quad \text { for all } u \leq 0 .
$$

This implies that $\bar{\delta}$ given by 2.2 is equal to $+\infty$, and therefore, Assumption 3.1 is satisfied for any initial stock position $y>0$. In other words, if we consider an exponential Lévy model and use the approximation scheme discussed above, we do not need to be concerned by any restriction on the maximum volume being liquidated.

With $L$ given by $(5.8)$ and $\hat{L}$ defined in Proposition 5.2 , in view of $(3.26)-(3.27)$ we consider the optimization problem

$$
V(y)=\inf _{Y \in \mathcal{A}_{D}(y)} \int_{0}^{\infty}\left(\hat{\kappa}_{\tilde{A}}\left(Y_{t}\right)+A \xi_{t} F\left(\xi_{t}\right)\right) d t, \quad y \geq 0,
$$

where $A>0$ denotes the investor's risk aversion, $\tilde{A}=A \tilde{s}$ and $\hat{\kappa}_{\tilde{A}}:[0, \infty) \rightarrow[0, \infty)$ is defined by $\hat{\kappa}_{\tilde{A}}(x)=\hat{\kappa}(-\tilde{A} x)$ with $\hat{\kappa}$ being the cumulant generating function of $\hat{L}_{1}$.

Theorem 5.4. The unique optimal liquidation speed for problem (5.25) is given by

$$
\xi_{t}^{*}=G\left(\frac{\hat{\kappa}_{\tilde{A}}\left(Y_{t}^{*}\right)}{A}\right), \quad t \geq 0,
$$

where $G:[0, \infty) \rightarrow[0, \infty)$ is the inverse function of $x \mapsto x^{2} F^{\prime}(x)$ and $Y^{*}$ is the associated unique optimal admissible stock position process satisfying

$$
\int_{Y_{t}^{*}}^{y} \frac{1}{G\left(\frac{\hat{\kappa}_{\tilde{A}}(u)}{A}\right)} d u=t, \quad \text { if } t \leq \tau, \quad \text { and } \quad Y_{t}^{*}=0, \quad \text { if } t>\tau,
$$

with $\tau$ defined by

$$
\tau=\int_{0}^{y} \frac{1}{G\left(\frac{\kappa_{A}(u)}{A}\right)} d u .
$$

The value function in (5.25) satisfies

$$
V(y)=\int_{0}^{y}\left\{\frac{\hat{\kappa}_{\tilde{A}}(u)}{G\left(\frac{\hat{\kappa}_{\tilde{A}}(u)}{A}\right)}+A F\left(G\left(\frac{\hat{\kappa}_{\tilde{A}}(u)}{A}\right)\right)\right\} d u, \quad y \geq 0 .
$$

Proof. This is a direct consequence of Theorem 4.3 . 


\section{$6 \quad$ Examples}

In this section, we provide some examples following the approximation scheme discussed in the previous section. We consider the process $\tilde{L}$ in $(5.1)$ as a variance-gamma (VG) Lévy process, which is obtained by subordinating a Brownian motion using a gamma process.

Let $\Gamma(a, b)$ denote the gamma distribution with shape parameter $a>0$ and rate parameter $b>0$, for which the probability density function is given by $f(x)=\frac{b^{a}}{\Gamma(a)} x^{a-1} \mathrm{e}^{-b x}$, for $x>0$, where $\Gamma(\cdot)$ is the gamma function. For any $X \sim \Gamma(a, b)$, it is known that $\mathbb{E}[X]=\frac{a}{b}$ and $\operatorname{Var}[X]=\frac{a}{b^{2}}$. The gamma process with parameters $(\gamma, \lambda)$ is then the pure jump Lévy process with Lévy-measure

$$
\nu(d z)=\frac{\gamma}{z} \mathrm{e}^{-\lambda z}, \quad z>0
$$

and zero otherwise. The VG Lévy process with parameters $(\rho, \eta, \theta)$ is then defined as follows. Let $W$ denote a standard Brownian motion, and let $\tau$ denote the gamma process with parameters $(1, \eta)$, that is $\tau$ is the pure jump Lévy process such that $\tau_{t} \sim \Gamma\left(\frac{t}{\eta}, \frac{1}{\eta}\right)$. Then the VG Lévy Process $\tilde{L}$ is given by

$$
\tilde{L}_{t}=\theta \tau_{t}+\rho W_{\tau_{t}}, \quad t \geq 0 .
$$

The Lévy density of the VG Lévy process $\tilde{L}$ is then given by

$$
\tilde{f}(z)=\frac{1}{\eta|z|} \mathrm{e}^{C z-D|z|}, \quad z \in \mathbb{R}
$$

where

$$
C=\frac{\theta}{\rho^{2}} \quad \text { and } \quad D=\frac{\sqrt{\theta^{2}+\frac{2 \rho^{2}}{\eta}}}{\rho^{2}},
$$

and its cumulant generating function $\tilde{\kappa}$ admits the expression

$$
\tilde{\kappa}(x)=-\frac{1}{\eta} \ln \left(1-\frac{x^{2} \rho^{2} \eta}{2}-\theta \eta x\right)
$$

(Cont and Tankov, 2004). It can be shown that Assumption 5.1 is satisfied if $D-C>2$. With reference to Proposition 5.2 , we calculate that the Lévy measure $\hat{\nu}$ of the process $\hat{L}$ satisfies

$$
\hat{\nu}(d x)= \begin{cases}\frac{-1}{\eta \ln (x+1)}(x+1)^{C+D-1} d x, & x \in(-1,0), \\ \frac{1}{\eta \ln (x+1)}(x+1)^{C-D-1} d x, & x \in(0, \infty) .\end{cases}
$$


Therefore, the function $\hat{\kappa}_{\tilde{A}}:[0, \infty) \rightarrow[0, \infty)$ in $(5.25)$, denoting it by $\hat{\kappa}_{\tilde{A}}^{V G}$ in the example of VG Lévy process, is given by

$$
\hat{\kappa}_{\tilde{A}}^{V G}(u)=-\tilde{A} \tilde{m} u+\int_{-1}^{\infty}\left(\mathrm{e}^{-\tilde{A} u x}-1+\tilde{A} u x\right) \hat{\nu}(d x),
$$

where the drift parameter $\tilde{m}=\tilde{\kappa}(1)$.

The next result provides a lower bound for $\hat{\kappa}_{\tilde{A}}^{V G}$, which later will be useful for deciding the limit behaviour of the price impact function.

Proposition 6.1. For $u \geq 0$, write

$$
\begin{aligned}
\underline{\hat{\kappa}}_{\tilde{A}}^{V G}(u)=-\tilde{A} \tilde{m} u+\frac{\mathrm{e}}{\eta}\left[-\frac{\mathrm{e}^{\tilde{A} u} \tilde{A} u}{C+D+2}\left(\frac{1}{\tilde{A} u} \wedge 1\right)^{C+D+2}+\frac{\mathrm{e}^{\tilde{A} u}}{C+D+1}\left(\frac{1}{\tilde{A} u} \wedge 1\right)^{C+D+1}\right. \\
\left.+\frac{\tilde{A} u}{C+D+2}-\frac{1+\tilde{A} u}{C+D+1}\right]
\end{aligned}
$$

and in particular, for $u \geq \frac{1}{\tilde{A}}$,

$$
\begin{gathered}
\underline{\hat{\kappa}}_{\tilde{A}}^{V G}(u)=-\tilde{A} \tilde{m} u+\frac{\mathrm{e}}{\eta}\left[\left(\frac{1}{\tilde{A} u}\right)^{C+D+1} \mathrm{e}^{\tilde{A} u}\left(\frac{1}{C+D+1}-\frac{1}{C+D+2}\right)\right. \\
\left.+\frac{\tilde{A} u}{C+D+2}-\frac{1+\tilde{A} u}{C+D+1}\right] .
\end{gathered}
$$

Then we have $\hat{\kappa}_{\tilde{A}}^{V G}(u) \geq \underline{\hat{\kappa}}_{\tilde{A}}^{V G}(u)$, for all $u \geq 0$.

Proof. For $u \geq 0$, we calculate that

$$
\begin{aligned}
& \int_{-1}^{0}\left(\mathrm{e}^{-\tilde{A} u x}-1+\tilde{A} u x\right) \hat{\nu}(d x) \\
= & \int_{-1}^{0}\left(\mathrm{e}^{-\tilde{A} u x}-1+\tilde{A} u x\right) \frac{-1}{\eta \ln (x+1)}(x+1)^{C+D-1} d x \\
\geq & \frac{\mathrm{e}}{\eta} \int_{-1}^{0}\left(\mathrm{e}^{-\tilde{A} u x}-1+\tilde{A} u x\right)(x+1)^{C+D} d x \\
= & \frac{\mathrm{e}}{\eta} \int_{0}^{1}\left(\mathrm{e}^{-\tilde{A} u(x-1)} x^{C+D}\right) d x+\frac{\mathrm{e}}{\eta} \int_{0}^{1}\left(\tilde{A} u x^{C+D+1}\right) d x+\frac{\mathrm{e}}{\eta} \int_{0}^{1}\left(-(1+\tilde{A} u) x^{C+D}\right) d x
\end{aligned}
$$

where the first inequality is due to $\frac{-1}{(x+1) \ln (x+1)} \geq \mathrm{e}$, for all $-1<x<0$, since $(x+1) \ln (x+1)$ 
is convex with minimum value $-\mathrm{e}^{-1}$. Observe that

$$
\begin{aligned}
& \int_{0}^{1}\left(\mathrm{e}^{-\tilde{A} u(x-1)} x^{C+D}\right) d x \\
\geq & \mathrm{e}^{\tilde{A} u} \int_{0}^{\frac{1}{\tilde{A} u} \wedge 1}\left((-\tilde{A} u x+1) x^{C+D}\right) d x \\
= & -\frac{\tilde{A} u \mathrm{e}^{\tilde{A} u}}{C+D+2}\left(\frac{1}{\tilde{A} u} \wedge 1\right)^{C+D+2}+\frac{\mathrm{e}^{\tilde{A} u}}{C+D+1}\left(\frac{1}{\tilde{A} u} \wedge 1\right)^{C+D+1}
\end{aligned}
$$

and

$$
\int_{0}^{1}\left(\tilde{A} u x^{C+D+1}\right) d x+\int_{0}^{1}\left(-(1+\tilde{A} u) x^{C+D}\right) d x=\frac{\tilde{A} u}{C+D+2}-\frac{1+\tilde{A} u}{C+D+1}
$$

where we have $C+D>0$, and the inequality is because $\mathrm{e}^{-\tilde{A} u x} \geq-\tilde{A} u x+1$ on the interval $\left[0, \frac{1}{\tilde{A} u} \wedge 1\right]$. Therefore, the required result follows from $6.10-6.12$ and the expression for $\hat{\kappa}_{\tilde{A}}^{V G}$ in 6.7) as well as the fact that $\mathrm{e}^{-\tilde{A} u x}-1+\tilde{A} u x$ and $\hat{\nu}$ are positive for all $u \geq 0$ and $x \in \mathbb{R}$.

In order to compare the optimal strategy for the model involving a VG Lévy process and the optimal strategy for the corresponding model with a Brownian motion (i.e. when $\tilde{L}$ is a Brownian motion), we derive that the function $\hat{\kappa}_{\tilde{A}}:[0, \infty) \rightarrow[0, \infty)$ in $(5.25)$, denoting it by $\hat{\kappa}_{\tilde{A}}^{B M}$, is given by

$$
\hat{\kappa}_{\tilde{A}}^{B M}(u)=-\tilde{A}\left(\tilde{\mu}+\frac{\tilde{\sigma}^{2}}{2}\right) u+\frac{1}{2} \tilde{A}^{2} \tilde{\sigma}^{2} u^{2}
$$

where $\tilde{\mu} \in \mathbb{R}$ and $\tilde{\sigma}>0$ are some constants which represent the drift and volatility of $\tilde{L}$, respectively. For this case, Assumption 5.1 is always satisfied.

Throughout this section we use the following parameters for our VG Lévy process: $\theta=$ $-0.002, \rho=0.02$ and $\eta=0.6$ (timescale in days). For more details on empirical studies of parameters of the VG stock price model we refer to Rathgeber et al. (2013). For parameters in the Brownian motion case, we choose the parameters such that the expectation and the second moment of $\mathrm{e}^{\tilde{L}_{t}}$ match that of the VG model. Hence, $\tilde{\mu}$ and $\tilde{\sigma}$ in 6.13 are taken to be such that $\tilde{\mu}+\frac{\tilde{\sigma}^{2}}{2}=\tilde{\kappa}(1)$ and $2 \tilde{\mu}+2 \tilde{\sigma}^{2}=\tilde{\kappa}(2)$, where $\tilde{\kappa}$ is given by 6.5 . Therefore, throughout this section,

$$
\tilde{\mu}=2 \tilde{\kappa}(1)-\frac{\tilde{\kappa}(2)}{2} \quad \text { and } \quad \tilde{\sigma}^{2}=\tilde{\kappa}(2)-2 \tilde{\kappa}(1) .
$$

Moreover, we choose $\tilde{s}=100$ for simplicity. 


\subsection{Power-law price impact function}

Consider the power-law temporary impact function, i.e. $F:[0, \infty) \rightarrow[0, \infty)$ is given by

$$
F(x)=\beta x^{\gamma},
$$

where $\beta>0$ and $\gamma>0$ are constants. This kind of impact function is widely believed to be realistic and has been well-studied in the literature of price impact (Lillo et al. , 2003), (Almgren et al., 2005). It can be checked that $F$ satisfies Assumption 2.3, and the function $G$ appearing in $(5.26)$ is given by

$$
G(x)=\left(\frac{x}{\beta \gamma}\right)^{\frac{1}{\gamma+1}}, \quad x \geq 0 .
$$

Applying Proposition 4.4. we see that if $\hat{L}$ is a strict supermartingale, then $\tau$ in 4.8 is finite, for all $\gamma>0$; if $\hat{L}$ is a martingale, then $\tau=\infty$ for $\gamma \in(0,1]$, and $\tau<\infty$ when $\gamma>1$. It follows from (5.26) that the optimal liquidation speed takes the expression

$$
\xi_{t}^{*}=\left(\frac{\hat{\kappa}_{\tilde{A}}\left(Y_{t}^{*}\right)}{A \beta \gamma}\right)^{\frac{1}{\gamma+1}}, \quad \text { for all } t \geq 0 .
$$

We adopt the values of $\beta$ and $\gamma$ suggested in Almgren et al. (2005) where parameters of the power-law temporary impact are studied empirically. In particular, we take $\gamma=0.6$ and $\beta=4.7 \times 10^{-5}[$

Consider a stock with average daily volume $2 \times 10^{6}$. Suppose the investor wants to liquidate a position of $2 \times 10^{5} 2^{2}$ of this stock. Figure 11 shows the optimal liquidation trajectories for both the VG Lévy process case and the Brownian motion case when the risk aversion parameter $A$ takes values of $10^{-6}, 10^{-5}$ and $10^{-4}$. [3 We see that when $A=10^{-6}$, the

\footnotetext{
${ }^{1}$ With our notations, the temporary impact function $F$ in Almgren et al. (2005) is given by $F(x)=\beta x^{\gamma}=$ $\tilde{S}_{0-} \tilde{\beta} \tilde{\sigma}\left(\frac{x}{\tilde{V}}\right)^{\gamma}$, where $\tilde{V}$ denotes the daily volume of a given stock, the value of exponent $\gamma$ is argued to be 0.6 (as the main result in their paper) and $\tilde{\beta}$ is a constant which is suggested to be 0.142 . From the values of parameters of the VG Lévy process that we have chosen, it can be calculated that the volatility $\tilde{\sigma}$ in the Brownian motion case is roughly equal to 0.02. Comparing this number to the values of volatilities and daily volumes of stocks provided in examples in Almgren et al. $(2005)$, we may take $\tilde{V}=2 \times 10^{6}$ as a reasonable choice. Moreover, we choose $\tilde{s}=100$ for simplicity. Then $\beta$ is calculated to be $4.7 \times 10^{-5}$.

Note that the empirical study in Almgren et al. (2005) is based on a model parametrised by the volume time which is defined as fractions of a daily volume. Therefore, any results of number regarding time derived from a model with power-law impact function in this section should be interpreted as volume time.

${ }^{2}$ Since the study of parameters of impacts in Almgren et al. (2005) is based on liquidating the amount of shares that weighted as $10 \%$ of daily volume, in order to keep consistent with the values of parameters of the temporary impact function that we have chosen, we let the initial stock position to be $2 \times 10^{5}$ which is $10 \%$ of the daily volume that we have chosen as explained before.

${ }^{3}$ In view of the general literature on preferences, these values of $A$ may seem small. However, in the context of a liquidation model they can viewed as reasonable if the investor is not sensitive to any large costs which are insignificant compared to his total wealth. We refer to Almgren and Chriss (2000) and Almgren (2003) for more details about the risk aversion parameter for the Almgren-Chirss liquidation model.
} 

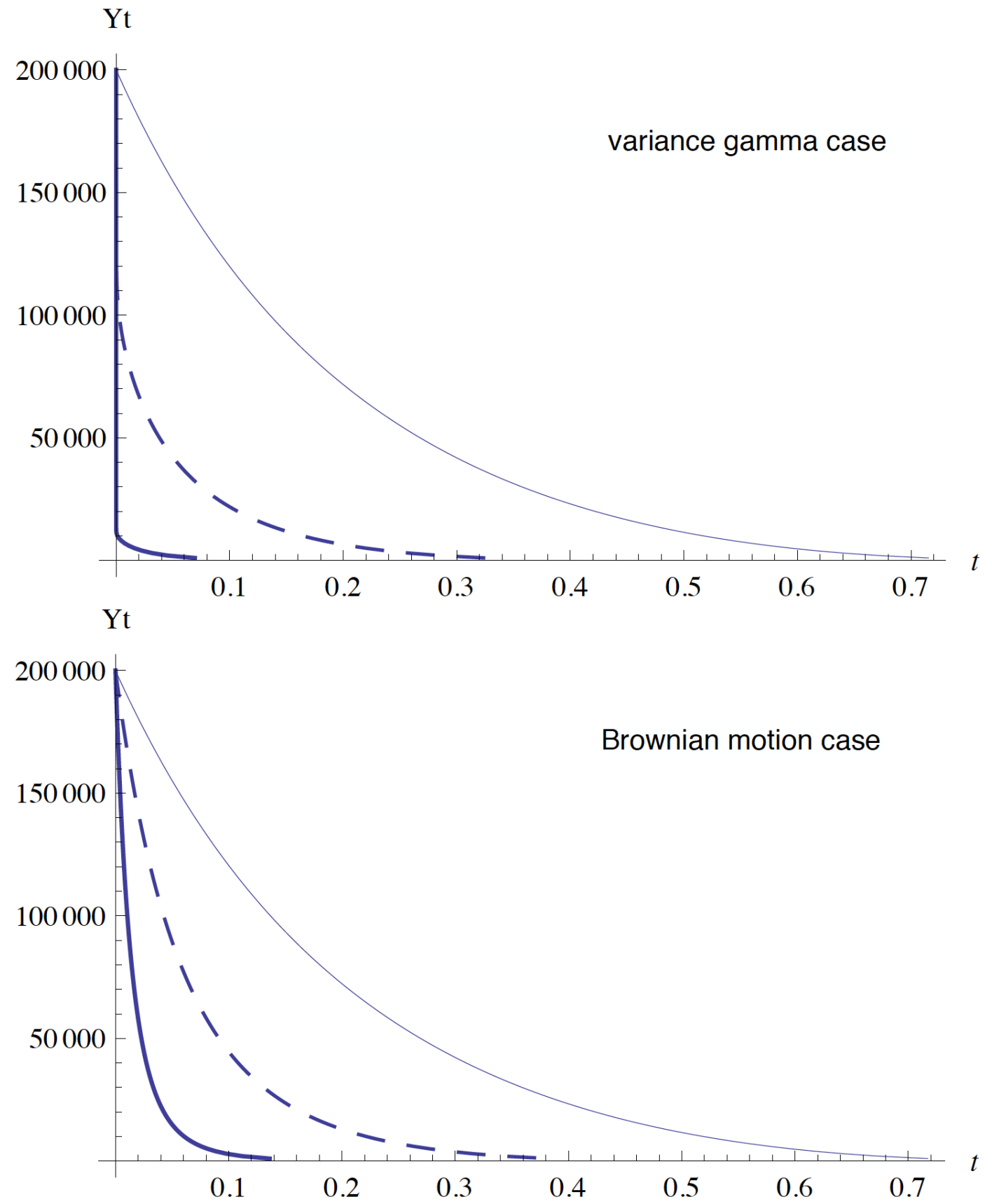

Figure 1: Optimal liquidation trajectories for variance-gamma Lévy process model and Brownian motion model with 0.6 power-law temporary impact function. Thin curves are for $A=10^{-6}$, dashed curves are when $A=10^{-5}$ and thick curves are for $A=10^{-4}$. 
optimal strategies for the two models are almost identical. As $A$ increases, the optimal speeds increase in both models, and in particular, speeds increase much faster in the VG model for large positions. In each case, liquidation finishes in a short time period, which confirms that the linear approximation of the exponential model is reasonable.

As shown in the first graph of Figure 1 when $A=10^{-5}$ and $A=10^{-4}$, the stock positions drop immediately by a large proportion of its initial value. In order to get more details about these two trajectories, we compute that when $A=10^{-5}$ the time spent on liquidating $40 \%$ of $2 \times 10^{5}$ shares is about 0.00018 when the investor follows the optimal strategy for the VG case. If the time parametrisation is the same as clock time, then 0.00018 is just a few seconds. If the investor's risk aversion is $A=10^{-4}$, then according to the optimal strategy for VG model, he spends roughly $1.34 \times 10^{-14}$ amount of time to liquidate $90 \%$ of his initial position.

With a large stock position, due to the nature of jumps of the VG Lévy process we expect that the investor would liquidate much faster than the optimal strategy for Brownian motion model. However, the above examples show that with the 0.6 power-law temporary impact function, in the VG case, optimal liquidation speeds can be too large for the optimal strategy to be practical, while speeds in the Brownian motion model stay in a reasonable range. Intuitively, an unrealistically high optimal liquidation speed can be due to price impact for large trading speeds being underestimated. In other words, the estimated cost resulting from large speeds being too small. This argument can be confirmed by the expression for the optimal liquidation speed in (5.26), that if the temporary impact function $F$ has a small growth rate then the growth rate of the function $G$ is large, and therefore the optimal speed can be very high when the stock position is large. It is mentioned in Roşu (2009) and in Gatheral (2010) that the impact function should be concave for small trading speeds and convex for large speeds.

\subsection{A relation between the impact functions of various models}

In this section we derive a connection between a temporary impact function for the Lévy liquidation model and a temporary impact function for the Brownian motion liquidation model such that the two respective optimal strategies coincide with each other.

Let $F^{L}:[0, \infty) \rightarrow[0, \infty)$ and $F^{B M}:[0, \infty) \rightarrow[0, \infty)$ be the temporary impact functions satisfying Assumption 2.3 considered in the Lévy model and the Brownian motion model, respectively. We denote by $G^{L}:[0, \infty) \rightarrow[0, \infty)$ and $G^{B M}:[0, \infty) \rightarrow[0, \infty)$ the inverse functions of $x \mapsto x^{2}\left(F^{L}\right)^{\prime}(x)$ and $x \mapsto x^{2}\left(F^{B M}\right)^{\prime}(x)$. Then in view of (5.26), the optimal liquidation speed at time $t$ for each model, denoted by $\xi_{t}^{L}$ and $\xi_{t}^{B M}$, is given by

$$
\xi_{t}^{L}=G^{L}\left(\frac{\hat{\kappa}_{\tilde{A}}^{L}\left(Y_{t}^{L}\right)}{A}\right) \quad \text { and } \quad \xi_{t}^{B M}=G^{B M}\left(\frac{\hat{\kappa}_{\tilde{A}}^{B M}\left(Y_{t}^{B M}\right)}{A}\right),
$$

where $\hat{\kappa}_{\tilde{A}}^{L}$ and $\hat{\kappa}_{\tilde{A}}^{B M}$ represents $\hat{\kappa}_{\tilde{A}}$ for the two different models, and $Y^{L}$ and $Y^{B M}$ are the corresponding optimal liquidation strategies in each model. Suppose that $Y_{t}^{*}=Y_{t}^{L}=Y_{t}^{B M}$, 
for all $t \geq 0$. Then

$$
G^{L}\left(\frac{\hat{\kappa}_{\tilde{A}}^{L}\left(Y_{t}^{*}\right)}{A}\right)=G^{B M}\left(\frac{\hat{\kappa}_{\tilde{A}}^{B M}\left(Y_{t}^{*}\right)}{A}\right), \quad t \geq 0
$$

Set $z=G^{B M}\left(\frac{\hat{\kappa}_{\tilde{A}}^{B M}\left(Y_{t}^{*}\right)}{A}\right)$. By 6.13 we have

$$
Y_{t}^{*}=\frac{\tilde{u}+\sqrt{\tilde{u}^{2}+2 A \tilde{\sigma}^{2} z^{2}\left(F^{B M}\right)^{\prime}(z)}}{\tilde{A} \tilde{\sigma}^{2}}
$$

where $\tilde{u}=\tilde{\mu}+\frac{\tilde{\sigma}^{2}}{2}$. Then from 6.19 we obtain that

$$
\left(F^{L}\right)^{\prime}(z)=\frac{1}{A z^{2}} \hat{\kappa}_{\tilde{A}}^{L}\left(\frac{\tilde{u}+\sqrt{\tilde{u}^{2}+2 A \tilde{\sigma}^{2} z^{2}\left(F^{B M}\right)^{\prime}(z)}}{\tilde{A} \tilde{\sigma}^{2}}\right),
$$

which is equivalent to

$$
F^{L}(x)=\int_{0}^{x} \frac{1}{A z^{2}} \hat{\kappa}_{\tilde{A}}^{L}\left(\frac{\tilde{u}+\sqrt{\tilde{u}^{2}+2 A \tilde{\sigma}^{2} z^{2}\left(F^{B M}\right)^{\prime}(z)}}{\tilde{A} \tilde{\sigma}^{2}}\right) d z .
$$

It can be shown that Assumption 2.3 is satisfied by the above expression. We can therefore conclude that if $F^{L}$ and $F^{B M}$ satisfy 6.22 , then $Y^{L}=Y^{B M}$.

Suppose $F^{B M}$ in 6.22 follows a power-law such that the optimal speed for the Brownian motion case is practically realistic (this kind of model is indeed used in practice). Then it follows from the relation in (6.22) that in order for the optimal speed in VG case to be practically realistic, the function $F^{L}$ needs to increase to infinity faster than any power function. This is because for the VG Lévy process case, the lower bound of the function $\hat{\kappa}_{\tilde{A}}^{V G}$ given in Proposition 6.1 tends to infinity faster than any power function.

\section{Conclusion}

For a market agent with constant absolute risk aversion, we have derived an explicit expression for the optimal liquidation trajectory when the unaffected stock price process follows a Lévy process and where the temporary impact function satisfies some reasonable assumptions. We have shown how to linearize exponential Lévy models to obtain approximations for our Lévy model, and we have shown that for the variance-gamma model the optimal liquidation trajectory is more rapid than for the standard Brownian motion model. We have also derived an explicit expression for the temporary impact function for the Lévy model that results in the same optimal liquidation trajectory as the optimal trajectory for the Brownian motion model. For the future it would be interesting to investigate if there are any connection between the distribution of the returns for a stock and the temporary impact function for 
that stock. Madan and Seneta (1990) showed that for the exponential variance-gamma Lévy model there is a strong connection between the subordinator and the volume traded. So for the variance-gamma model there is a connection between the distribution of the returns and the distribution of the volume traded. As liquidity is related to variability of the volume traded, a connection between the distribution of the returns and the temporary impact function seems plausible, but needs further investigation.

\section{References}

Alfonsi, A., Fruth, A., and Schied, A. (2010). Optimal execution strategies in limit order books with general shape functions. Quantitative Finance, 10:143-157.

Alfonsi, A. and Schied, A. (2010). Optimal trade execution and absence of price manipulations in limit order book models. SIAM Journal on Financial Mathematics, 1:490-522.

Alfonsi, A., Schied, A., and Slynko, A. (2012). Order book resilience, price manipulation, and the positive portfolio problem. SIAM Journal on Financial Mathematics, 3:511-533.

Almgren, R. (2003). Optimal execution with nonlinear impact functions and trading-enhanced risk. Risk, 10:1-18.

Almgren, R. and Chriss, N. (1999). Value under liquidation. Risk, 12(12):61-63.

Almgren, R. and Chriss, N. (2000). Optimal execution of portfolio transactions. Risk, 3(2):539.

Almgren, R., Thum, C., Hauptmann, E., and Li, H. (2005). Direct estimation of equity market impact. Risk, 18(7):58-62.

Barndorff-Nielsen, O. E. (1997). Normal inverse gaussian distribution and stochastic volatility modelling. Scandinavian Journal of Statistics, 24(1):1-13.

Bertsimas, D. and Lo, A. (1998). Optimal control of execution costs. Journal of Financial Markets, 1:1-50.

Cartea, A., Jaimungal, S., and Penalva, J. (2015). Algorithmic and High-Frequency Trading. Cambridge University Press.

Cont, R. and Tankov, P. (2004). Financial modelling with jump processes. Chapman \& Hall/CRC.

Eberlein, E. and Keller, U. (1995). Hyperbolic distributions in finance. Bernoulli, 1(3):281299. 
Forsyth, P. A., Kennedy, J. S., Tse, S. T., and Windcliff, H. (2012). Optimal trade execution: a mean-quadratic variation approach. Journal of Economic Dynamics and Control, 36(12):1971-1991.

Gatheral, J. (2010). No-dynamic-arbitrage and market impact. Quantitative Finance, 10(7):749-759.

Gatheral, J. and Schied, A. (2011). Optimal trade execution under geometric brownian motion in the Almgren and Chriss framework. International Journal of Theoretical and Applied Finance, 14:353-368.

Guéant, O. (2016). The Financial Mathematics of Market Liquidity. Chapman and Hall.

Guo, X. and Zervos, M. (2015). Optimal execution with mulitplicative price impact. SIAM Journal on Financial Mathematics, 6(1):281-306.

Kallenberg, O. (2001). Foundations of modern probability. Springer.

Kallsen, J. and Shiryaev, A. (2002). The cumulant process and Esscher's change of measure. Finance and Stochastics, 6:397-428.

Kyprianou, A. (2006). Introductory lectures on fluctuations of Levy processes with applications. Springer.

Lehalle, C.-A. and Laruelle, S. (2013). Market Microstructure in Practice. World Scientific.

Lillo, F., Farmer, J. D., and Mantegna, R. N. (2003). Econophysics - master curve for priceimpact function. Nature, 421(6919):129-130.

Løkka, A. (2014). Optimal liquidation in a limit order book for a risk-averse investor. Mathematical Finance, 24(4):696-727.

Madan, D. and Seneta, E. (1990). The variance gamma (V.G.) model for share market returns. The Journal of Business, 63(4):511-524.

Obizhaeva, A. and Wang, J. (2013). Optimal trading strategy and supply/demand dynamics. Journal of Financial Markets, 16:1-32.

Rathgeber, A., Stadler, J., and Stöckl, S. (2013). Modeling share returns - an empirical study on the variance gamma model. Proceedings of European Financial Management Association, Reading 2013.

Roşu, I. (2009). A dynamic model of the limit order book. Review of Financial Studies, 22:4601.

Rogers, L. C. G. and Williams, D. (2000). Diffusions, Markov processes and martingales. Cambridge University Press. 
Schied, A. (2013). Robust strategies for optimal order execution in the Almgren-Chriss framework. Applied Mathematical Finance, 20(3):264-286.

Schied, A. and Schöneborn, T. (2009). Risk aversion and the dynamics of optimal liquidation strategies in illiquid markets. Finance and Stochastics, 13:181-204.

Schied, A., Schöneborn, T., and Tehranchi, M. (2010). Optimal basket liquidation for CARA investors is deterministic. Applied Mathematical Finance, 17:471-489.

Schöneborn, T. (2016). Adaptive basket liquidation. Finance and Stochastics, 20:455-493. 